\title{
Large-to-small scale frequency modulation analysis in wall-bounded turbulence via visibility networks
}

\author{
Giovanni Iacobello $^{1} \dagger$, Luca Ridolfi ${ }^{2}$, and Stefania Scarsoglio ${ }^{1}$ \\ ${ }^{1}$ Department of Mechanical and Aerospace Engineering, Politecnico di Torino, Turin, Italy \\ ${ }^{2}$ Department of Environmental, Land and Infrastructure Engineering, Politecnico di Torino, Turin, Italy
}

(Received xx; revised xx; accepted xx)

Scale interaction is studied in wall-bounded turbulence by focusing on the frequency modulation (FM) mechanism of large scales on small scale velocity fluctuations. Differently from amplitude modulation analysis, frequency modulation has been less investigated also due to the difficulty to develop robust tools for broadband signals. To face this issue, the natural visibility graph approach is proposed in this work to map the full velocity signals into complex networks. We show that the network degree centrality is able to capture the signal structure at local scales directly from the full signal, thereby quantifying FM. Velocity signals from numerically-simulated turbulent channel flows and an experimental turbulent boundary layer are investigated at different Reynolds numbers. A correction of Taylor's hypothesis for time-series is proposed to overcome the overprediction of near-wall frequency modulation obtained when local mean velocity is used as the convective velocity. Results provide networkbased evidences of the large-to-small FM features for all the three velocity components in the near-wall region, with a reversal mechanism emerging far from the wall. Additionally, scaling arguments in the view of the quasi-steady quasi-homogeneous hypothesis are discussed, and a delay-time between large and small scales very close to the near-wall cycle characteristic time is detected. Results show that the visibility graph is a parameter-free tool that turns out to be effective and robust to detect FM in different configurations of wall-bounded turbulent flows. Based on present findings, the visibility network-based approach can represent a reliable tool to systematically investigate scale interaction mechanisms in wall-bounded turbulence.

\section{Introduction}

The characterization and modelling of wall-bounded turbulent flows is of paramount importance in physics and engineering (Marusic et al. 2010). Organized motions, in particular, play a crucial role in wall-bounded turbulence analysis, since they are associated to high energy levels and are directly involved in transport processes, making them preferential targets for flow control strategies (Jiménez 2018). Coherent streaks are recognized as the dominant flow structures very close to the wall, and are characterized by a distinctive (inner) peak in the spectrogram of the streamwise velocity fluctuations, $u$, within the buffer layer (Jiménez 2018). The investigation of high Reynolds number experiments and simulations also revealed the formation of large scale motions (LSMs) and very large scale motions (VLSMs) residing in the log-region (Smits et al. 2011), whose presence is detected by the appearance of another (outer) peak in the (pre-multiplied) energy spectrogram of the streamwise velocity fluctuations (Hutchins \& Marusic 2007b; Monty et al. 2009; Peruzzi et al. 2020). The wallnormal location in wall units (i.e., made dimensionless by the mean friction velocity, $U_{\tau}$, and the fluid kinematic viscosity, $v), y^{+}=y U_{\tau} / v$, of the inner peak is conventionally assumed

$\dagger$ Email address for correspondence: giovanni.iacobello@ polito.it 


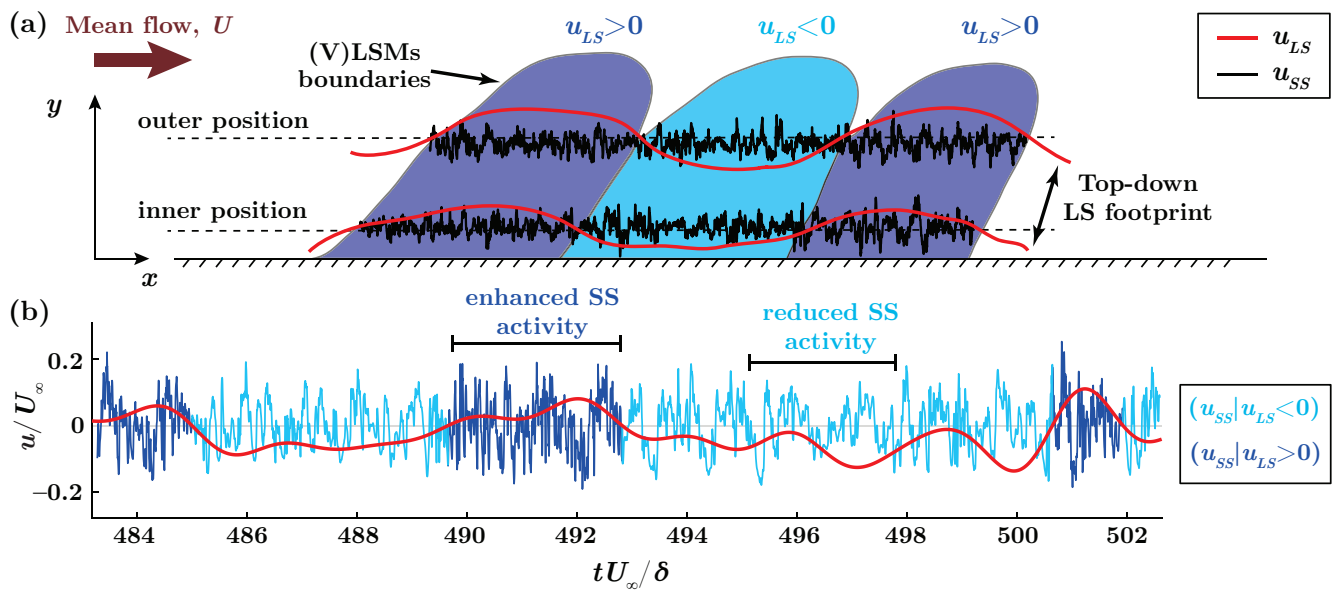

Figure 1: (a) Schematic of a wall-bounded turbulent flows in the $(x-y)$ plane, showing three alternating LSM and VLSM structures of uniform large scale momentum, $u_{L S} \lessgtr 0$. Two pairs of time-series of $u_{L S}$ and $u_{S S}$ are also depicted as red and black lines, respectively, at two wall-normal locations (referred to as inner and outer position). (b) The small scale, $u_{S S}$, and the large scale, $u_{L S}$, of streamwise velocity fluctuations at $y^{+} \approx 10$ are shown as blue and red lines, respectively, where light and dark blue portions of $u_{S S}$ correspond to intervals of $u_{L S}<0$ and $u_{L S}>0$, respectively. The velocity series is extracted from an experimental turbulent boundary layer at $R e_{\tau}=14750$ (Marusic 2020) in the range $t U_{\infty} / \delta=483-503$, where $U_{\infty}$ and $\delta$ are the free stream velocity and boundary layer thickness, respectively. Two intervals of the signal (i.e., $490<t U_{\infty} / \delta<493$ and $495<t U_{\infty} / \delta<498$ ) in which small scales display enhanced or reduced activity are also indicated.

to be fixed at $y^{+}=15$, while the position of the outer peak increases with the frictional Reynolds number, $R e_{\tau}$, as $y^{+} \approx 3.9 \operatorname{Re}_{\tau}^{1 / 2}$ (Mathis et al. 2009a).

Besides the effect of the Reynolds number, some differences emerged from the comparison of different canonical wall-bounded turbulent flows. While near-wall statistics (such as the mean velocity profile) agree well in channel, pipe and boundary layer flows, the features of large scales depend on the flow configuration (Monty et al. 2007; Balakumar \& Adrian 2007; Mathis et al. 2009b; Monty et al. 2009; Chernyshenko 2020). In particular, spectral analyses of internal and external flows have revealed that very-large scales tend to be longer for channel and pipe flows than boundary layer flows, although they appear to be qualitatively similar (Balakumar \& Adrian 2007; Monty et al. 2009). Such large scale differences are expected to grow for larger Reynolds numbers as energetic contributions coming from very-large scale motion increases with the Reynolds number.

The investigation of higher Reynolds number data has progressively opened novel developments and questions about an interaction between small scale turbulence (whose spectral peak occurs in the wall proximity) and large scale motions (whose spectral peak resides far from the wall). First insights on scale interaction were initially reported by Brown \& Thomas (1977) and Bandyopadhyay \& Hussain (1984), who observed a modulation mechanism of (nearwall) small scales by large turbulent scales. Later on, Hutchins \& Marusic (2007b) provided further evidences of a top-down footprint and an amplitude modulation (AM) phenomenon by the large scale motions residing in the log-region on the near-wall (small scale) dynamics. Aiming to illustrate such inter-scale mechanism, Figure 1(a) shows a schematic of a wallbounded turbulent flow in a streamwise-vertical plane, where uniform momentum regions due to LSM and VLSM (highlighted as dark and light blue structures) entail large scale fluctuations, $u_{L S}$ (see red lines). Figure 1(a) is drawn following the current picture of the kinematics of turbulent scales and their interaction in wall-bounded turbulence (e.g., 
see Ganapathisubramani et al. 2012; Baars et al. 2017). The turbulent flow field can be decomposed as $u(x, y, z, t)=u_{L S}(x, y, z, t)+u_{S S}(x, y, z, t)$, where $u_{S S}$ are small scale fluctuations (see black signals), while $x, y, z$ are the streamwise, vertical and spanwise directions, respectively, and $t$ is time. Figure 1(a) also highlights the top-down footprint of large scales, being the two $u_{L S}$ signals (red lines) positively correlated with each other (eventually accounting for the inclination of large scales (Marusic \& Heuer 2007)).

A modulation of the amplitude of the small scales caused by the large scales implies that high or low values of $u_{L S}$ correspond to (on average) high or low values of $u_{S S}$. This mechanism can be observed in Figure 1(b), which shows a time interval of $u_{L S}$ and $u_{S S}$ at $y^{+} \approx 10$ in an experimental turbulent boundary layer. An increase of the local amplitude of the small scale signal (see dark blue intervals) is discernible during positive large scale velocity fluctuations, $u_{L S}>0$, and, vice versa, a damping of small scale amplitudes (light blue intervals) during negative large scale velocity fluctuations, $u_{L S}<0$.

Mathis et al. (2009a) quantified this amplitude modulation by correlating $u_{L S}$ with the large-scale-filtered envelope of $u_{S S}$ at different wall-normal coordinates. The authors evidenced an amplitude modulation (as shown in Figure 1(b)) only close to the wall (approximatively below the center of the log-region), while a reversed AM mechanism i.e., an $u_{S S}$ amplitude increase under $u_{L S}<0$ and an $u_{S S}$ amplitude decrease under $u_{L S}>0$ - occurs far from the wall. Further studies on turbulent boundary layers have suggested that a modulation mechanism does actually take place only in the near-wall region, while different mechanisms occur in the log- and wake regions. In particular, the behaviour of scale interaction away from the wall has been explained either through a preferential arrangement of the small scales - i.e., an alignment of the small scale turbulence with internal shear layers that separate zones of large scale uniform momentum (Hutchins 2014; Baars et al. 2017) - or as an effect of variations in the mean strain and in the shear-driven production (Agostini \& Leschziner 2019).

Based on the insights from Hutchins \& Marusic (2007b) and Mathis et al. (2009a), AM has been largely investigated for several flow configurations and Reynolds numbers, both experimentally (e.g., see Mathis et al. 2009b; Schlatter \& Örlü 2010; Guala et al. 2011; Ganapathisubramani et al. 2012; Talluru et al. 2014; Baars et al. 2015; Duvvuri \& McKeon 2015; Squire et al. 2016; Baars et al. 2017; Pathikonda \& Christensen 2017; Basley et al. 2018; Pathikonda \& Christensen 2019) and numerically (e.g., see Chung \& McKeon 2010; Bernardini \& Pirozzoli 2011; Agostini \& Leschziner 2014; Hwang et al. 2016; Agostini et al. 2016; Anderson 2016; Yao et al. 2018; Dogan et al. 2019; Agostini \& Leschziner 2019). Furthermore, findings on scale interaction have fostered the development of predictive models for near-wall turbulence that explicitly account for the footprint and amplitude modulation by large scales on small scales (Marusic et al. 2010; Mathis et al. 2011, 2013; Baars et al. 2016; Wu et al. 2019). It should be noted that, although large-scale spectral features do not match between internal and external flows, similar AM results have been found for channel, pipe and boundary layer flows at similar $R e_{\tau}$ values (Mathis et al. 2009b), suggesting a similar scale-interaction mechanism is at play in all configurations.

Besides amplitude modulation, small scale turbulence has also been found to change its instantaneous (i.e., local) frequency during intervals of positive or negative $u_{L S}$, namely large scales affect the smalls scales through a frequency modulation (FM) mechanism (Ganapathisubramani et al. 2012; Fiscaletti et al. 2015; Baars et al. 2015). However, much less investigations to quantify FM in wall-bounded turbulence have been carried out so far (Ganapathisubramani et al. 2012; Baars et al. 2015, 2017; Pathikonda \& Christensen 2017; Tang \& Jiang 2018; Awasthi \& Anderson 2018; Pathikonda \& Christensen 2019) if compared with the vaster literature on amplitude 
modulation and its application into predictive models. One of the main reasons for this literature imbalance resides on the difficulty to produce robust methodologies to quantify FM in broadband signals, as well as the difficulty to effectively capture instantaneous frequencies in a signal.

Aiming to quantify FM in the context of wall-bounded turbulence scale interaction, two methodologies have been proposed as yet. Ganapathisubramani et al. (2012) proposed a peak-valley approach, following the idea that local frequency is proportional to the number of maxima and/or minima per unit length of the series. The peak-valley approach was applied to streamwise $u_{S S}$ signals from experimental measurements in a turbulent boundary layer at $\operatorname{Re}_{\tau}=\delta U_{\tau} / v=14150$ (where $\delta$ is the boundary layer thickness). Similarly to AM, the authors found a relevant FM of the small scales in the near-wall region in which higher frequencies correspond to large (positive) $u_{L S}$ values while lower frequencies correspond to low (negative) $u_{L S}$ values. However, differently from AM, substantial FM was observed only up to $y^{+} \approx 100$. As an example of this FM mechanism, in Figure $1(\mathrm{~b})$ a rapidly fluctuating $u_{S S}$ activity can be seen during positive $u_{L S}$ (dark blue intervals) than negative $u_{L S}$ (light blue intervals). Despite its conceptual simplicity, the main drawback of the peak-valley approach is the need of a signal discretization into sub-intervals of arbitrary spacing to quantify the number of maxima and minima within each sub-interval. The choice of the size of the signal partition into sub-intervals is non-trivial and requires a trade-off between too short or too large intervals that can affect the results. Moreover, as pointed out by Baars et al. (2015), the short-time partitioning of the peak-valley approach makes it less applicable if temporal shifts in amplitude and frequency modulation have to be focused.

An alternative approach to quantify FM and effectively account for time shifts was then proposed by Baars et al. (2015), who exploited wavelet analysis to extract from the velocity time-series a new signal that is representative of the local frequency variations at the small scales. The authors performed a time-frequency analysis of the streamwise velocity, in which a time-series - representative of the small scale instantaneous frequency - was obtained by evaluating the first spectral moment of the wavelet power spectrum, namely an average energetic contribution at each time coming from the range of (high) frequencies pertaining the small scales (Baars et al. 2015). The first spectral moment was eventually long-wavelength pass filtered to retain only its large scale component, and correlated with $u_{L S}$ to quantify FM (similarly to the AM technique proposed by Mathis et al. (2009a)).

The wavelet-based procedure was applied to experimental streamwise velocity time-series measured at different wall-normal locations from a turbulent boundary layer at $R e_{\tau}=$ 14750 (Baars et al. 2015). The authors showed positive correlations up to the center of the log-region, meaning that higher and lower frequencies in $u_{S S}$ are detected under $u_{L S}>0$ and $u_{L S}<0$, respectively. Almost zero correlations were observed, instead, for higher wallnormal locations up to the boundary layer intermittent region, where negative correlation values were detected. The near-wall FM found by Baars et al. (2015) is in accordance with the outcomes from Ganapathisubramani et al. (2012), but the $y^{+}$coordinate above which FM was found to be almost absent is larger by using the wavelet-based approach $\left(y^{+} \approx 470\right)$ than the peak-valley approach $\left(y^{+} \approx 100\right)$, although the $\operatorname{Re}_{\tau}$ values were rather similar. Furthermore, a phase lead of the small scale amplitude and frequency was found in the near-wall with respect to the large scale signals, and - in accordance with previous studies (Bandyopadhyay \& Hussain 1984; Guala et al. 2011) - a much larger lead was detected for small scale amplitudes than for frequency. Although the FM has been accepted as a near-wall mechanism, it is still not fully clear the interaction mechanism in terms of FM between small and large scales in the log- and wake regions, in particular what is the precise wall-normal coordinate at which small scale frequency is no longer affected by large scales. 
So far, the wavelet-based technique by Baars et al. (2015) has been exploited as the main tool to quantify FM in wall-bounded turbulence. Different flow configurations have been explored in terms of FM, such as experimental smooth-wall turbulent boundary layers via hot-wire measurements (Baars et al. 2017) and particle image velocimetry (Pathikonda \& Christensen 2019), experimental boundary layers in presence of wall roughness (Pathikonda \& Christensen 2017; Tang \& Jiang 2018), as well as large eddy simulation of a turbulent channel flow with spanwise heterogeneity (Awasthi \& Anderson 2018). These works highlighted that - despite the specific quantitative differences - near-wall FM is present both for smooth- and rough-walls, as well as for several Reynolds numbers. However, despite its preferred employment for quantifying FM, the wavelet-based approach presents some criticalities. First, as discussed by Baars et al. (2015), the choice of the mother wavelet can have an impact on the results since different frequency resolutions are gained from different mother wavelets. Moreover, the procedure necessitates multiple filtering operations that demand the choice of an appropriate frequency filter value. In particular, a frequency threshold is required both in the computation of the first spectral moment of the wavelet power spectrum (that involves a numerical integration), and in the long-wavelength pass filtering of the first spectral moment. Therefore, differently from the peak-valley approach by Ganapathisubramani et al. (2012) - in which maxima and minima are counted - the wavelet-based approach intrinsically requires several procedural steps and assumptions that need to be carefully handled.

In this work, a novel approach to study FM in wall-bounded turbulence is put forward with a twofold aim: (i) to propose a non-parametric and robust methodology to extract local frequency changes in a signal, and (ii) to show its effectiveness for two wall-bounded turbulence configurations, also reporting novel insights that can help to further shed light on large-small scale interaction. Our methodology relies on the natural visibility graph (NVG) approach proposed by Lacasa et al. (2008), which is used to map a signal into a network by exploiting a geometrical criterion. Thanks to its simplicity of implementation, the NVG has been widely employed in a large variety of research areas such as, among many others, economy, biomedicine, geophysics (Zou et al. 2018). In particular, visibility-based investigations have been carried out in fluid mechanics to study jets and fires (Charakopoulos et al. 2014; Murugesan et al. 2019; Tokami et al. 2020), wall-bounded turbulent flows (Liu et al. 2010; Iacobello et al. 2018b), passive scalar plumes (Iacobello et al. 2018a, 2019a), and turbulent combustors (Murugesan \& Sujith 2015, 2016; Singh et al. 2017).

In spite of its simplicity, the NVG approach (defined in $\S 2.1$ ) has been shown to be a powerful tool in capturing important features of the mapped signal (such as the occurrence of extreme events) and a reliable indicator of the transition between different flow dynamics (Iacobello et al. 2021). Here we show that the degree centrality - which is one of the simplest network metrics - is much more sensitive to the small scale spectral energy variations than the large scale counterpart (2.2). Accordingly, the network degree is viewed as a metric that is able to inherit the local frequency variations in a signal (2.3), without any a priori assumption (e.g., signal filtering). Therefore, the NVG approach can be directly used to study the full velocity signals rather than the small scale component.

The proposed NVG approach is used to analyse time-series (\$ 3.2) from an experimental smooth-wall zero-pressure-gradient turbulent boundary layer $\left(R e_{\tau}=14750\right.$, Marusic 2020), and spatial-series - namely 1D signals along spatial transects at fixed time (\$ 3.1) - from two direct numerical simulations (DNSs) of smooth-wall incompressible turbulent channel flows $\left(R e_{\tau} \approx 5200\right.$ and $R e_{\tau}=1000$, Lee \& Moser 2015; Graham et al. 2016). In this regard, for simplicity, we refer to as FM to indicate both temporal and spatial frequency (i.e., wavenumber) modulation, where the former applies to time-series while the latter to spatialseries. A comparative FM analysis is performed by highlighting differences and similarities 
between outcomes from the two wall-bounded turbulence setups for the streamwise velocity (§ 4.1). In particular, the effect of different Reynolds numbers is examined, and the application of Taylor's hypothesis to time-series is discussed by proposing a convection velocity that compensates for overprediction of modulation in the near-wall region. Moreover, FM results are examined in the view of the quasi-steady quasi-homogeneous theory, in terms of degree centrality scaling with respect to large scale velocity values $(\S 4.2)$. The analysis is then extended to the wall-normal and spanwise velocities of the channel flow (\$ 4.3), and time and space shifting are eventually investigated for all the three velocity components $(\$ 4.4)$. Finally, we provide a discussion on some general features of the visibility approach $(\S 5)$ as well as concluding remarks $(\$ 6)$.

\section{Visibility-based analysis of frequency modulation}

\subsection{Definition of visibility graph}

Visibility graphs represent a widely employed technique to map a discrete signal in a network. The idea behind the visibility graph approach is to assign a node of the network to each datum in the signal, and activate a link between two nodes if a geometrical criterion is satisfied. The main variant is the natural visibility graph (NVG), which is based on a convexity criterion (Lacasa et al. 2008). Geometrically, two nodes in an NVG (corresponding to two points in the signal) are linked if the straight line connecting the two points lies above any other in-between data. Figure 2(a, lower diagram) shows an example of a short series, $s_{i} \equiv s\left(\chi_{i}\right)$, for the independent variable $\chi_{i}$ (i.e., a time or space coordinate), comprising $N=20$ observations, illustrated as vertical bars. Nodes and links in Figure 2(a) are depicted as filled circles at the tip of each bar and green straight lines, respectively. A representative node is highlighted in red and its links are reported in orange.

The NVG criterion applied to a generic signal, $s(\chi)$, can be formally written as:

$$
s\left(\chi_{n}\right)<s\left(\chi_{j}\right)+\left(s\left(\chi_{i}\right)-s\left(\chi_{j}\right)\right) \frac{\chi_{j}-\chi_{n}}{\chi_{j}-\chi_{i}}, \quad i, j=1, \ldots, N,
$$

for any $\chi_{n}$ (i.e., time or space coordinate) such that $\chi_{i}<\chi_{n}<\chi_{j}$ (Lacasa et al. 2008). The corresponding visibility network is represented through the adjacency (binary) matrix $\boldsymbol{A}$, whose entries are $A_{i, j}=1$ if the inequality (2.1) is satisfied for the node pair $(i, j)$ with $i \neq j$, and $A_{i, j}=0$ otherwise. For example, in Figure 2(a), the node $i=8$ is connected (i.e., $A_{8, j}=1$ ) to nodes $j=\{1,2,3,4,5,7,9\}$, as highlighted by the orange links. By definition, visibility networks are connected (i.e., each node $i$ is linked to at least one other node $j$, e.g., $j=i+1$ or $j=i-1$ ) and undirected (Newman 2018), namely the adjacency matrix is symmetric $\left(A_{i, j}=A_{j, i}\right)$.

Differently from other techniques developed to transform a signal into a network (Zou et al. 2018; Iacobello et al. 2021), the visibility algorithm does not require any a priori parameter. Given a signal, a unique visibility network is obtained in a straightforward way by applying the convexity criterion in (2.1) for each pair of data. Another feature of NVGs is the invariance under affine transformations of the mapped signal, namely translation and rescaling (i.e., multiplication by a positive constant) of both horizontal and vertical axes (Lacasa et al. 2008). This implies that two signals with the same temporal (or spatial) structure but with different mean values (i.e., vertical translation of the series) and standard deviations (i.e., vertical rescaling of the series) are mapped in the same visibility graph.

In the present work, we exploited the NVG approach to study turbulent velocity signals from wall-bounded turbulence, both as time-series (from the boundary layer, § 3.2) and spatialseries (from the channel flow, § 3.1). We note that this is the first time the NVG is employed 
(a)

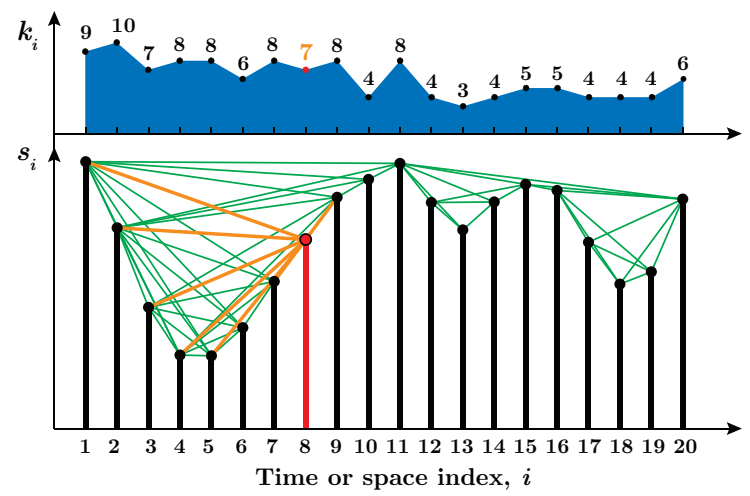

(b)

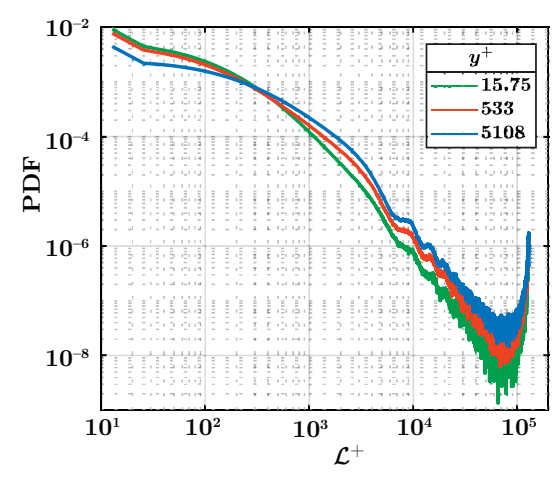

Figure 2: (a) The lower diagram shows an example of a signal, $s_{i} \equiv s\left(\chi_{i}\right)$, and the corresponding visibility network, where nodes are depicted as black filled circles and links as green lines. In particular, the node $i=8$ and its links are highlighted in orange. The degree values for each node, $k_{i}$, are also shown in the upper diagram. (b) PDF of the link length evaluated on the network built from streamwise velocity, $u(x)$, in a turbulent channel flow at $R e_{\tau} \approx 5200$. The link-length is expressed in wall-units as $\mathcal{L}^{+}=|i-j| \Delta x^{+}$, where $i$ and $j$ are the indices of two connected nodes and $\Delta x^{+}=12.7$ (see $\S 3.1$ ).

for studying wall-bounded turbulence by focusing on spatial-series rather than time-series. An optimized code for computing the NVG (either for spatial- and time-series) was provided by Iacobello (2020), where the possibility to account for spatial-series periodicity is also implemented.

One remarkable feature of NVGs from signals referring to physical phenomena with a wide range of different scales (such as in turbulence) is the infrequent appearance of longrange links. In fact, the presence of fluctuations of different amplitude in the signal prevents the possibility that a node is visible by other distant nodes (Zhuang et al. 2014). To grasp the concept, the probability density function (PDF) of the link length in $u(x)$ signals from a turbulent channel flow $\left(R e_{\tau} \approx 5200\right.$, see $\left.\S 3.1\right)$ is shown in Figure 2(b), evidencing that long-range links are very unlikely to occur (the increasing PDF for large $\mathcal{L}^{+}$values is due to signal periodicity in the $x$-direction).

The capability of visibility graphs to capture the temporal (or spatial) structure of a signal by means of a convexity-based geometrical framework, hence, turns out to be a key feature to study the occurrence in time (or space) of specific events (Iacobello et al. 2018a, 2019a). In this work, we take advantage from the features of visibility networks to detect frequency modulation of large scales on small scales.

\subsection{Node degree in relation to small scale signal features}

The degree centrality (or, simply, degree) of a node, $i$, is defined as the number of neighbours of $i$, that is the number of nodes linked to $i$,

$$
k_{i} \equiv \sum_{j=1}^{N} A_{i, j},
$$

where $N$ is the total number of nodes, corresponding to the number of sampled values of the signal (Newman 2018). The top panel in Figure 2(a) shows the sequence of degree values for the example of signal, $s_{i}$, shown in the bottom of Figure 2(a); for instance, the degree of node $i=8$ (highlighted in red) is $k_{8}=7$ since it is connected to seven other points (links are highlighted in orange). By averaging over all nodes, a representative degree value 
(a)

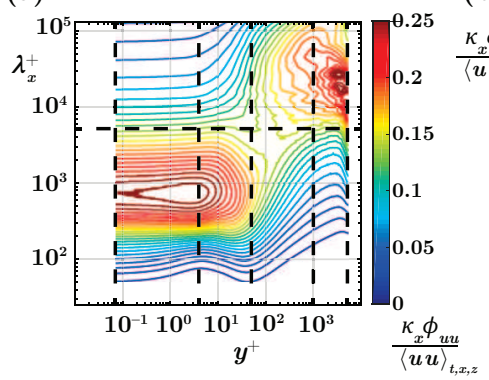

(b)

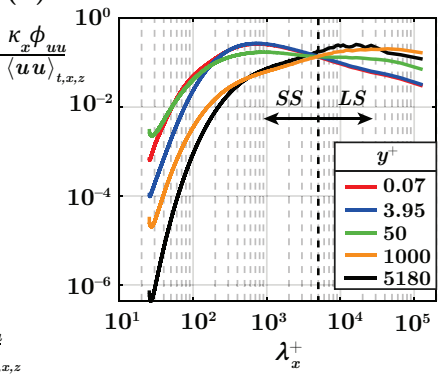

(c)

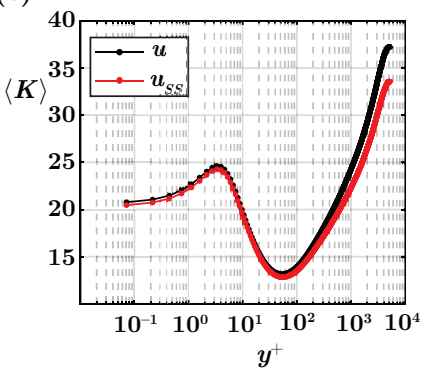

Figure 3: (a) Pre-multiplied energy spectral density, $\phi_{u u}$, from a turbulent channel flow at $R e_{\tau} \approx 5200$ (see $\S 3.1$ ), normalized by the streamwise velocity variance, $\langle u u\rangle_{t, x, z}$. The horizontal dashed line indicates the value of spectral filter, while vertical dashed lines highlight five representative $y^{+}$coordinates. (b) Premultiplied energy spectral density for the five selected $y^{+}$locations in (a). (c) Wall-normal behaviour of the average degree centrality, $\langle K\rangle$, for NVG built from the full streamwise velocity, $u\left(x_{i}\right)$ (black curve), and from the small scale streamwise velocity, $u_{S S}\left(x_{i}\right)$ (red curve), obtained through a spectral decomposition. Angular brackets in $\langle K\rangle$ indicate averaging over the (homogeneous) spanwise direction, $z$.

for the network (i.e., for the whole signal) is obtained as $K=\sum_{i} k_{i} / N$. It should be noted that the degree, $k_{i}$, provides a measure of the extent to which a single node $i$ belongs to a convex interval in the signal, but it is not directly able to quantify whether the properties of node $i$ (e.g., its importance in the network) are similar or not to the properties of other nodes. Instead, this issue can be tackled through assortativity measures, which can be used to assess similarities among nodes (e.g., in terms of their importance in the network through degree-degree correlation) (Newman 2018).

Recalling that long-range links unlikely appear in visibility graphs (Figure 2(b)), the main contribution to degree values is due to short-range links, making the degree a metric that is typically sensitive to the local structure of the signal. Rapidly fluctuating signals are then expected to show lower degree values, $k_{i}$, and in turn a lower average degree $K$ (Zhuang et al. 2014). Since rapid variations in the local structure of turbulent signals are mainly governed by high frequencies (i.e., low wavelengths), a relation should exist between the average degree, $K$, and the high-frequency spectral energy (that produces the local variations in the turbulent signals).

With the aim to explore this relation, we report in Figure 3(a) the energy spectral density of the streamwise velocity, $\phi_{u u}$, pre-multiplied for the wavenumber, $\kappa_{x}=2 \pi / \lambda_{x}$, from a turbulent channel flow at $R e_{\tau} \approx 5200$ (see $\S 3.1$ ). Notice that $\phi_{u u}$ is normalized by the variance of the streamwise velocity fluctuations, $\langle u u\rangle_{t, x, z}$ (here angular brackets indicate the average over time, $t$, and homogeneous directions, $x, z)$. In Figure 3(a) it is easily distinguishable the spectral peak separation between small and large scales, as well as the spectral filter adopted in this work, marked as a horizontal dashed line. Five curves of the spectrum at five representative $y^{+}$coordinates (highlighted as dashed vertical lines in Figure 3(a)) are also shown in Figure 3(b).

The rationale behind the normalization of the spectrum through the variance is twofold: on the one side, the streamwise energy density at each $y^{+}$is accentuated, thus emphasizing the occurrence of the two spectral peaks and, on the other side, this normalization permits a congruent comparison with the degree behaviour computed on visibility networks (which are insensitive to different variance levels, i.e., on signal rescaling). Moreover, due to the variance normalization, the area under each curve in Figure 3(b) is equal to unity, so that the integral of curves in Figure 3(b) in a given range of $\lambda_{x}$ represents the fraction of total energy pertaining that scale range. In this way, Figure $3(\mathrm{~b})$ elucidates the redistribution of 
the spectral energy density over scales, $\lambda_{x}^{+}$, at different $y^{+}$coordinates (a log-log plot in Figure 3(b) is shown with the aim to highlight the behaviour at small $\lambda_{x}^{+}$values).

Focusing on the small scales (say, $\lambda_{x}^{+}<R e_{\tau} \approx 5200$ ) in Figure 3(b), we observe that by moving from very close to the wall $\left(y^{+} \approx 0.07\right)$ up to $y^{+} \approx 4$ there is a small decrease in the (normalized) energy content, then an increase of the (normalized) spectral energy occurs from $y^{+} \approx 4$ up to the beginning of the log-layer $\left(y^{+} \approx 50\right)$, and lastly a persistent decrease happens up to the channel centreline $\left(y^{+} \approx 5200\right)$. A reduction or a growth of the (normalized) spectral energy at small scales indicates that the signal tends to be locally smoother (i.e., slowly-varying, without rapid low-intensity fluctuations) or more irregular (i.e., rapidly-varying), respectively. Recalling that the mean degree, $K$, is sensitive to the local structure of the signal, an increase of the degree values is then expected for locally smoother signals (i.e., low spectral energy at local scales), and vice versa. Figure 3(c) shows the wall-normal behaviour of the mean degree, $K$, of networks built from the full streamwise velocity, $u\left(x_{i}\right)$ (black line), in the channel flow setup. As expected, the $y^{+}$-trend of $K$ for the full signal closely follows the behaviour of the small scale spectral energy density as described above, where the degree growth is faithfully related to the small scale spectralenergy decrease, and vice versa. In particular, we point out that the value of $K$ at each $y^{+}$ is associated to an integral effect of all wavelengths in the signal, so that $K\left(y^{+}\right)$is due to a cumulative effect of different spectral-energy levels.

Figure 3(c) also shows the $y^{+}$-behaviour of the mean degree of networks built from $u_{S S}\left(x_{i}\right)$ (red line), namely, in which the large scale component is removed. The values and the trends of $K$ from the full and the small scale velocity signals are very close, and a slight difference appears only very far from the wall. Note that a similar behaviour of $K$ as that shown in Figure 3(c) for the channel case is also found for the turbulent boundary layer case. It should be noted that, since very long-range connections are unlikely to appear (Figure 2(b, they only barely contribute to the average degree, $K$, which instead is mainly related to shorter links.

The very good agreement between $K\left(y^{+}\right)$for the full $u$ signal and the $y^{+}$-variations in the small scale spectral energy (corroborated by the similarity of $K\left(y^{+}\right)$for $u$ and $u_{S S}$ ) indicates that the network degree is able to capture the features of the small scale turbulence directly from the full signal, i.e. without the arbitrary requirements of filtering operations. These features will be exploited in the next Section 2.3 to provide a metric which is able to quantify frequency modulation. We notice that, to the best of our knowledge, this is the first time that insights from the visibility graph approach are directly related to spectral properties of a signal.

\subsection{FM detection via degree centrality}

The aim of this section is to provide a degree-based metric able to quantify FM from full velocity signals. With this aim, in Figure 4(b) we show a short representative interval of the streamwise velocity series reported in Figure 4(a), which is extracted from the turbulent channel flow at $y^{+} \approx 10$. The corresponding NVG is then built from the short signal in Figure 4(b), and the links activated by two representative nodes, $i=\{19,49\}$ (highlighted as red dots in Figure 4(b)), are shown as green arcs in Figure 4(c). Node $i=19$ clearly displays more connections than node $i=49$ (i.e., $k_{19}>k_{49}$ ), since node $i=19$ is in a larger convex interval than $i=49$; in other words, the signal around $i=49$ varies more rapidly than around $i=19$. Therefore, although the degree, $k_{i}$, represents a pointwise value because $k_{i}$ refers to a single coordinate $i$, the information enclosed in $k_{i}$ originates from the surroundings of $i$. The degree $k_{i}$ can then be interpreted as a measure of the instantaneous period (or instantaneous wavelength) at the temporal (or spatial) coordinate $t_{i}$ (or $x_{i}$ ), in analogy with the concept of instantaneous frequency used in signal analysis (Huang et al. 1998; Boashash 2015). Larger 
(a)

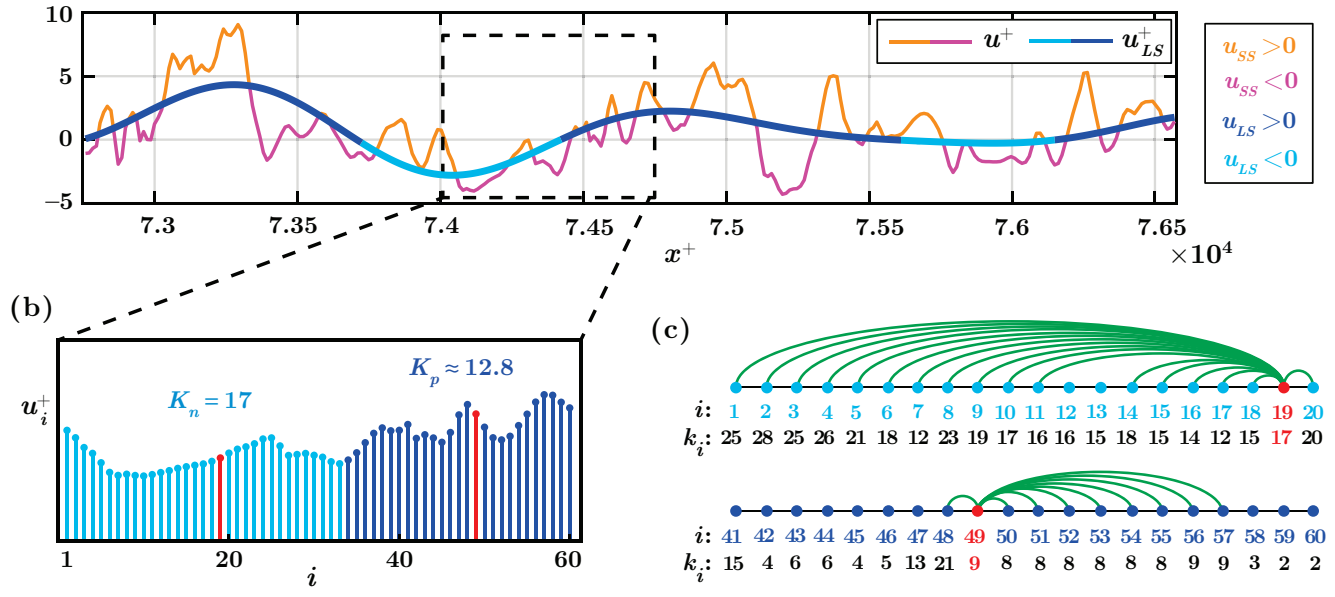

Figure 4: (a) An interval of the streamwise velocity, $u$, and its large scale component, $u_{L S}$, extracted along the streamwise direction, $x$, of the turbulent channel flow at $y^{+} \approx 10$. The full $u$ signal is depicted as orangemagenta lines indicating intervals of positive and negative small scale velocity fluctuations, $u_{S S}=u-u_{L S}$, respectively, while the $u_{L S}$ signal is depicted as light-dark blue lines, highlighting intervals of $u_{L S}<0$ and $u_{L S}>0$, respectively. Both the series are normalized in wall-units. (b) A piece consisting of 60 data of the velocity $u$ from panel (a), depicted as vertical bars whose color reflects the sign of $u_{L S}$. Two representative data (corresponding to nodes $i=19$ and $i=49$ of the NVG network) are highlighted in red. (c) Network representation of the NVG built from signal in panel (b). Two subsets of nodes and links from the two representative nodes, $i=\{19,49\}$, are shown as coloured dots and green arcs, respectively. The sequence of degree values, $k_{i}$, for each node, $i$, is also reported.

$k_{i}$ values correspond to larger instantaneous periods (or wavelengths), and in turn to smaller instantaneous frequencies.

On the basis of this argument and the insights illustrated in the previous Section 2.2, we introduce the ratio, $K_{n p}$, to quantify frequency modulation, defined as

$$
K_{n p} \equiv \frac{K_{n}}{K_{p}}, \quad K_{n}=\frac{1}{N_{n e g}} \sum_{j=1}^{N}\left(k_{j} \mid u_{L S}<0\right), \quad K_{p}=\frac{1}{N_{p o s}} \sum_{j=1}^{N}\left(k_{j} \mid u_{L S}>0\right),
$$

where $K_{n}$ and $K_{p}$ are the average degree values computed on the NVG of the full velocity signal, conditioned to intervals of $u_{L S}<0$ and $u_{L S}>0$, respectively, while $N_{\text {neg }}$ and $N_{\text {pos }}$ are the number of occurrences in which $u_{L S}<0$ and $u_{L S}>0$, respectively.

Values of $K_{n p}$ greater than 1 indicate that the degree is (on average) larger during $u_{L S}<0$ intervals than during during $u_{L S}>0$, and vice versa for $K_{n p}$ smaller than 1 . In the example of Figure 4(b-c), the degree values, $k_{i}$, of the two representative nodes in red $\left(k_{19}=17\right.$ and $k_{49}=9$ ) exemplify the behaviour of the two signal intervals during $u_{L S}<0$ and $u_{L S}>0$, resulting in $K_{n}=17, K_{p} \approx 12.8$ and $K_{n p}>1$. Hence, $K_{n p}$ discriminates between (i) positive frequency modulation for $K_{n p}>1$ (i.e., an increase of the local frequency in the velocity signal gained for $u_{L S}>0$ and a decrease for $u_{L S}<0$ ), (ii) negative frequency modulation for $K_{n p}<1$, and (iii) an absence of modulation for $K_{n p} \approx 1$. We emphasize that the arguments leading to the ratio (2.3) do not involve any a priori parameter, but the unique availability of the full velocity signal to compute the degree value of each node. A filtering operation is only required to condition the degree values to the sign of the large scale velocity.

To test the NVG-based approach, we built synthetic signals that mimic the near-wall 
modulation mechanism in wall-bounded turbulence for three modulation cases: amplitude modulation (AM), frequency modulation (FM), and both amplitude and frequency modulation. Appendix A contains details on the synthetic signal construction and reports the $K_{n p}$ values for each configuration (see Figure 9), showing that $K_{n p}$ is able to highlight the presence of FM and - in presence of both AM and FM mechanisms - tends to be more sensitive to FM while only weakly to AM.

In summary, the $K_{n p}$ ratio combines the capability of visibility networks (i) to capture the information on the local temporal structure of a series (§ 2.1), and (ii) to inherit the small scale energetic features from the full signal $(\$ 2.2)$. These characteristics make the visibility approach a powerful and easy-to-use alternative to previously proposed methodologies for time-frequency characterization of turbulence signals. In the following, the NVG-approach is carried out for wall-turbulent signals, showing its robustness (with respect to different cut-off filtering size) and effectiveness in capturing the large-to-small scale FM mechanism.

\section{Description of the turbulent flow datasets}

Two main datasets of high Reynolds number wall-bounded turbulent flows are exploited in this work to study frequency modulation by means of visibility network-based tools: (i) spatialseries from a numerically-simulated turbulent channel flow at $R e_{\tau} \approx 5200$ (Lee \& Moser 2015), and (ii) time-series from experimental measurements in a turbulent boundary layer at $R e_{\tau}=14750$ (Marusic 2020). Although outer flow structures start to occur and play a role in scale interaction at lower Reynolds numbers (Agostini \& Leschziner 2014; Hu \& Zheng 2018; Wu et al. 2019), high Reynolds number flows are required to enhance the inter-scale separation and amplify the scale interaction mechanism. Moreover, a third DNS dataset of turbulent channel flow at $R e_{\tau}=1000$ is also employed for comparison purposes, thus showing effects of inertial on FM results.

To the best of our knowledge, this is the first time a state-of-the-art DNS at $R e_{\tau} \approx 5200$ is employed to specifically investigate large-to-small scale FM. In fact, while high Reynolds number boundary layer flows are typically obtained in experimental facilities (as witnessed by most of previous works on $\mathrm{AM}$ and $\mathrm{FM}$ ), high- $R e_{\tau}$ experiments of channel flows are difficult to realise due to strong side-wall boundary effects (Lee \& Moser 2015). The DNS employed in this work is at a large enough Reynolds number (i.e., $R e_{\tau}>4000$, as reported by Hutchins \& Marusic 2007b) to guarantee a sufficient large-small scale spectral separation (e.g., see energy peaks separation in Figure 3(a)), and allows us to perform a FM analysis on all the three velocity components that, so far, has only been performed for AM (e.g., see Talluru et al. 2014; Agostini \& Leschziner 2016).

The scale decomposition of the streamwise velocity fluctuation signals was performed as $u(x)=u_{L S}(x)+u_{S S}(x)$ (e.g., Figure 4(a)) and $u(t)=u_{L S}(t)+u_{S S}(t)$ (e.g., Figure 1(b)) for the spatial- and time-series taken from the turbulent channel and boundary layer flows, respectively. A common approach to obtain $u_{S S}$ and $u_{L S}$ is to employ a spectral filter to retain the high and low wavelength or frequency, respectively, as performed in several previous works (Mathis et al. 2009a; Ganapathisubramani et al. 2012; Baars et al. 2015, 2017; Pathikonda \& Christensen 2017, 2019). Alternatively, Agostini \& Leschziner (2014) proposed to employ the empirical mode decomposition (Huang et al. 1998) to separate large and small scale contributions. In this work, both the spectral and empirical mode decompositions were tested to separate the large and small scale contributions. However, for the sake of simplicity and in line with most of the current literature, results are only shown for a spectral decomposition, as both the procedures produce equivalent results. 


\subsection{DNS of turbulent channel flows}

Velocity fields were extracted from two direct numerical simulations of incompressible turbulent channel flows. The first DNS was run at frictional Reynolds number $\operatorname{Re}_{\tau} \equiv$ $h U_{\tau} / v=5186$, where $h=1$ is the half-channel height, $U_{\tau}=4.14872 \times 10^{-2} U_{b}$ and $v=8 \times 10^{-6} U_{b} h$, with the bulk velocity $U_{b}=1$. The size of the spatial domain is $(8 \pi h \times 2 h \times 3 \pi h)$ with $(10240 \times 1536 \times 7680)$ grid points along the streamwise, wallnormal and spanwise directions, respectively. The flow fields were recorded only after statistical stationarity of the flow was reached, and 11 temporal frames of velocity and pressure spatial fields were stored in the dataset. The time interval between two consecutive frames is about 0.7 flow-through time, corresponding to about $3785 v / U_{\tau}^{2}$ in wall-units.

The simulation was performed at a sufficiently high Reynolds number and with a sufficiently large spatial domain to exhibit characteristics of high-Reynolds-number turbulence, e.g., the presence of large scale motions and a rather large wall-normal range for statistics scaling (Lee \& Moser 2015). The dataset is available online (doi:10.7281/T1PV6HJV) from the Johns Hopkins Turbulence Database (Li et al. 2008). For further simulation details and statistics, see Lee \& Moser (2015).

The second DNS was run at $R e_{\tau} \equiv h U_{\tau} / v=1000$, with $h=1, U_{\tau}=4.9968 \times 10^{-2} U_{b}, v=$ $5 \times 10^{-5} U_{b} h$ and $U_{b}=1$. The size of the spatial domain is $(8 \pi h \times 2 h \times 3 \pi h)$ with $(2048 \times 512 \times$ 1536) grid points along the streamwise, wall-normal and spanwise directions, respectively. Data were stored for approximately one flow-through time, $[0,26] h / U_{b}$, with a storage temporal step of 0.0065 . Also this dataset is available online (doi:10.7281/T10K26QW) from the Johns Hopkins Turbulence Database (Li et al. 2008). For further simulation details, see Graham et al. (2016).

In this work, 1D spatial-series (i.e., extracted at a fixed time) of the three velocity components, $u, v, w$, along the streamwise direction, $x$, are exploited to build visibility networks. Network-based results are averaged in time (i.e., on 11 temporal frames for the $R e_{\tau} \approx 5200$ setup, and on 400 uniformly spaced temporal frames for the $\operatorname{Re}_{\tau}=1000$ setup) and in the spanwise direction; in the latter case, averages are performed for a set of uniformly-spaced spanwise locations separated from each other by 64 and 128 grid points for the $R e_{\tau} \approx 5200$ and $R e_{\tau}=1000$ configurations, respectively.

The cut-off spectral filter to separate large and small scale streamwise velocity for the $R e_{\tau} \approx 5200$ setup is set equal to $\lambda_{x, c}=h$ (i.e., $\lambda_{x, c}^{+}=5186$ ), in analogy with previous works in which $\lambda_{x, c}$ is set equal to the boundary layer thickness (Hutchins \& Marusic 2007b; Mathis et al. 2009a,b; Marusic et al. 2010; Dogan et al. 2019; Wu et al. 2019). For the $R e_{\tau}=1000$ setup, the cut-off filter is $\lambda_{x, c}^{+}=5000$, thus being comparable with $\lambda_{x, c}^{+}$of the higher- $R e_{\tau}$ channel flow setup.

\subsection{Experimental turbulent boundary layer at $R e_{\tau} \approx 14750$}

Experimental measurements were performed in the wind-tunnel facility of the University of Melbourne, which employs a $27 \mathrm{~m}$ test section, under a free-stream velocity $U_{\infty}=$ $19.95 \mathrm{~m} / \mathrm{s}$ (Baars et al. 2015). Under these conditions, a zero-pressure-gradient boundary layer develops at a frictional Reynolds number $R e_{\tau} \equiv \delta U_{\tau} / v=14750$, where $\delta=0.361 \mathrm{~m}$ is the boundary layer thickness at the measuring location (i.e., $21.65 \mathrm{~m}$ from the inlet of the test section), while $U_{\tau}=0.626 \mathrm{~m} / \mathrm{s}$ and $v=1.532 \times 10^{-5} \mathrm{~m}^{2} / \mathrm{s}$ at the same streamwise location. The dataset is the same employed by Baars et al. $(2015,2017)$, and is available online at the Fluid Mechanics Research webpage of the University of Melbourne (Marusic 2020).

Time-series of the streamwise velocity were simultaneously recorded by means of two constant-temperature hot wire probes, one at a fixed wall-normal location at $y^{+}=4.33$, and the other vertically moved throughout the boundary layer in the range $y^{+} \in\left[10.5,2.14 \times 10^{4}\right]$ 
(or $y / \delta \in\left[7.087 \times 10^{-4}, 1.45\right]$ ) for 40 vertical locations. At each wall-normal measuring location, three sets of data are recorded at a sampling frequency of $20 \mathrm{kHz}$, each one for $120 \mathrm{~s}$ corresponding to a large scale time $6.6 \times 10^{3} \delta / U_{\infty}$, thus ensuring the convergence of spectral statistics at the longest energetic wavelengths (Baars et al. 2015). The resulting time-step in wall units is $\Delta t^{+}=1.28$. Further details on the measurement procedure and instrumentation can be found in Baars et al. (2015).

In order to separate large and small scale components, we employ a cut-off spectral filter $\lambda_{x, c}^{+}=7000$ following Hutchins \& Marusic (2007b), Hutchins (2014) and Baars et al. (2015, 2017), who showed this is a proper filter value for turbulent boundary layers at high Reynolds numbers. Differently from the channel flow setup in which spatial-series are considered, here the spectral filter is converted in terms of frequency by invoking the Taylor's hypothesis as $f_{c}\left(y^{+}\right)=U_{c}\left(y^{+}\right) / \lambda_{x, c}$, where $U_{c}\left(y^{+}\right)$is a local convection velocity at the wall-normal coordinate $y^{+}$. The effects of different convection velocities on FM will be elucidated in the next $\S 4$, where a comparison with spatial data from DNSs is carried out.

\section{Results}

The results of the application of the degree centrality as a metric to quantify frequency modulation are reported in this section, for velocity signals extracted from the turbulent channel flows and the turbulent boundary layer described above. A one-point modulation analysis is carried out: the large scale component, $u_{L S}$, used to condition the degree on the $u_{L S}$ sign (see equation 2.3) is extracted at the same $y^{+}$in which the signal is mapped into a visibility network. Due to the footprint of the large and very-large scale motions towards the wall, $u_{L S}$ evaluated at each $y^{+}$represents a good estimate of the large scale velocity component in the outer region, thus resulting in a more applicable procedure than two-points analysis (Mathis et al. 2009a). In fact, two-point synchronized measurements are not easy to perform experimentally (Mathis et al. 2009a). Previous works have shown that similar results are obtained by adopting a one- or two-point procedure for characterizing scales interaction (see, among others, Hutchins \& Marusic 2007a; Mathis et al. 2009a; Ganapathisubramani et al. 2012), thus one-point modulation is here preferred for simplicity.

First, the streamwise velocity component, $u$, is considered, both in an overall perspective $(\S 4.1)$ and with near-wall focus $(\S 4.2)$. Most of the current literature on scale interaction in wall-bounded turbulence is indeed focused on the $u$ component, being the component in which large and small scales can be clearly separated. We then extend the analysis to the other velocity components, $v$ and $w(\S 4.3)$. Finally, a space-shifted FM analysis is carried out for all velocity components and for both the turbulence configurations ( $\$ 4.4)$.

\subsection{FM in the streamwise velocity component}

The values of the ratio $K_{n p}$ as a function of the wall-normal coordinate, $y^{+}$, are shown in Figure 5 for visibility networks built from the streamwise velocity, $u$. We recall that $K_{n p}>1$ indicates a higher frequency under intervals of positive large scale velocity than under negative ones, and vice versa for $K_{n p}<1$.

Figure 5(a) shows $K_{n p}$ for the spatial-series of the two channel flow DNS at $\operatorname{Re}_{\tau} \approx 5200$ (black) and $R e_{\tau}=1000$ (red), while Figure 5(b) illustrates the $K_{n p}$ behaviour for timeseries of the boundary layer (green). Values of $K_{n p}>1$ are detected close to the wall for all configurations, while - moving away from the wall $-K_{n p}$ becomes smaller than 1 , indicating a reverse scale interaction mechanism, i.e., higher frequency are detected during $u_{L S}<0$ than under $u_{L S}>0$. The overall behaviours shown in Figure 5 are in accordance with previous works on scale interaction in wall-bounded turbulence, which have indicated that a higher (amplitude and) frequency of the small scales is found under positive $u_{L S}$ 
(a)

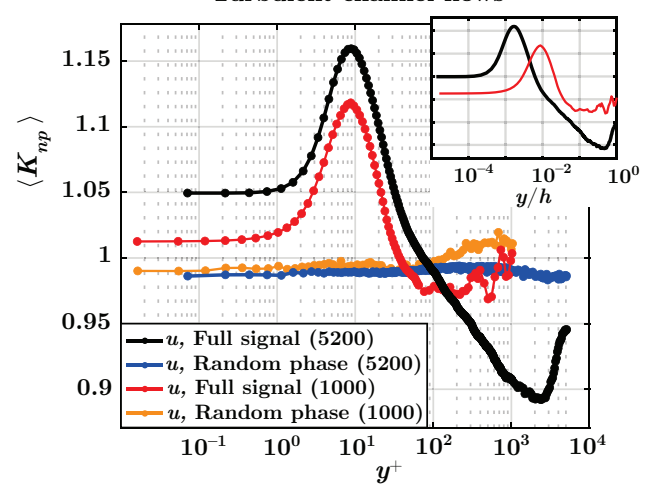

(b)

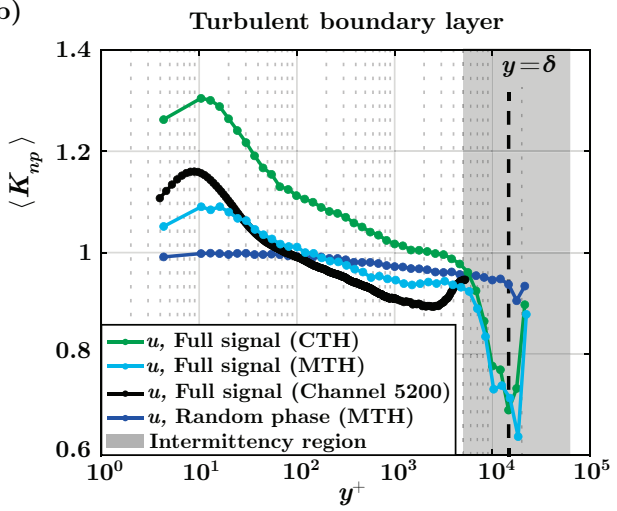

Figure 5: Large scale conditional average degree ratio, $K_{n p}$, as a function of the wall-normal coordinate, $y^{+}$, for streamwise velocity, $u$, extracted from (a) the channel flow DNS at different $R e_{\tau}$ and (b) the boundary layer experiment. The inset in (a) shows the $K_{n p}$ behaviour for the two channel flows as a function of $y / h$. In panel (b), $K_{n p}\left(y^{+}\right)$is shown for spatial-series obtained through the classical (CTH) and modified (MTH) Taylor's hypothesis as green and cyan plots, respectively; for comparison, the behaviour for the channel flow at $R e_{\tau} \approx 5200$ is also reported in black. Moreover, the boundary layer intermittency region is highlighted in (b) as a shaded grey region. Angular brackets indicate averaging over time and spanwise direction in (a) and over three different realizations in (b). The results for synthetic velocity signals with shuffled phases are also shown.

intervals in regions close to the wall, while a reversal mechanism occurs far from the wall (Ganapathisubramani et al. 2012; Baars et al. 2015, 2017; Pathikonda \& Christensen 2017; Tang \& Jiang 2018; Awasthi \& Anderson 2018; Pathikonda \& Christensen 2019). However, the behaviour of $K_{n p}\left(y^{+}\right)$for the two setups highlights also peculiar features of large-to-small scale FM that deserve further investigations.

First, we compare the results for the two channel flows at different Reynolds numbers (red and black lines in Figure 5(a)). A similar trend of $K_{n p}\left(y^{+}\right)$is found for both channels, but the intensity of the FM (close to the wall) is larger for $R e_{\tau} \approx 5200$ than for $R e_{\tau}=1000$, thus clearly showing the effect of higher Reynolds numbers is to increase FM mechanism in the near-wall region, as a consequence of increasing magnitude of the large-scale fluctuations with increasing $R e_{\tau}$. Similarly, away from the wall the reversal mechanism of scale interaction is strengthened for the higher Reynolds number DNS. Furthermore, in Figure 5(a) - and also in Figure 5(b) for the boundary layer - we observe a peak of $K_{n p}$ at $y^{+} \approx 10$ : this peak turns out to be related to strong sweep-like events, a phenomenon referred to as splatting in which large scales transport high-intensity small scales towards the wall below the buffer layer (Agostini \& Leschziner 2014; Agostini et al. 2016). Thus, being the highest small scale intensity detected in the buffer layer (Agostini et al. 2016), the strongest FM is revealed by a peak in $K_{n p}$, which then represents a sensitive metric to local changes in the flow dynamics. Eventually, it is remarkable to observe in Figure 5(a) the near-wall agreement of $K_{n p}$ plotted against $y^{+}$between the two channel flows at different Reynolds numbers, as the near-wall dynamics is related to near-wall cycle whose characteristic scales are fixed in wall-units (see also a discussion on characteristic near-wall spatial and temporal scales in $\S 4.4)$.

The behaviour of $K_{n p}$ obtained from time-series of the turbulent boundary layer is shown in Figure 5(b). When time-series are considered, a convection velocity, $U_{c}$, has to be defined to apply Taylor's hypothesis in filtering large and small scales. Typically, $U_{c}$ is set equal to the local mean velocity, $U$, and in the following this assumption will be referred to as classical Taylor's hypothesis (CTH). $K_{n p}$ as a function of $y^{+}$obtained through the CTH is displayed in green in Figure 5(b): although a similar behaviour with respect to the channel 
flows is observed (e.g., the black line in Figure 5(b)), there is a significant vertical shift when time-series are employed. It is worth noting that, since the local mean velocity, $U$, does not depend on time, the temporal structure of the time-series is preserved when classical Taylor's hypothesis is applied. This implies that the application of any technique (including NVG) to study scale-interaction from time-series is the same as from the corresponding spatial-series (i.e., obtained through the classical Taylor's hypothesis), being $\Delta x \propto \Delta t$.

The overestimation of modulation parameters when time-series and CTH are used has been previously observed for amplitude modulation in jet (Fiscaletti et al. 2015), mixing layer (Fiscaletti et al. 2016) and turbulent boundary layer flows (Yang \& Howland 2018). Specifically, Yang \& Howland (2018) reported a distortion of the spatial-series when the classical Taylor's hypothesis is used, and suggested to employ a convection velocity, $U_{c}$, defined as $U_{c}(t)=U+\alpha u(t)$, where $u(t)=u_{L S}(t)+u_{S S}(t)$ is the fluctuating component of the streamwise velocity, and $\alpha=O(1)$ is a proportionality constant. The correction proposed by Yang \& Howland (2018) is based on the rationale that the sampling time step has to be scaled using the local viscous scales, so that small scale activity is enhanced (or reduced) where the wall shear stress is high (or low) due to an increase (decrease) in the local friction velocity (Yang \& Howland 2018). However, variations in the (fluctuating) wall shear stress are mainly induced by variations into large scale fluctuations, $u_{L S}(t)$, rather than $u(t)$, as observed by Yang \& Howland (2018) and reported in previous literature (e.g., Zhang \& Chernyshenko 2016; Baars et al. 2017) (see a more detailed discussion about the relation between near-wall small scales and wall shear stress in $\S 4.2)$. Therefore, in this work we exploit the time-varying formulation by Yang \& Howland (2018) but only accounting for the large scale component of $u(t)$, namely

$$
U_{c}(t)=U+\alpha u_{L S}(t) .
$$

In what follows, we will refer to the application of (4.1) as the convection velocity as the modified Taylor's hypothesis (MTH), where we selected $\alpha=0.8$ (which has proved to be a suitable value). It should be noted that a correction based on $u_{L S}$ arguments was also discussed by Fiscaletti et al. (2015) for jet and boundary layer flows to compensate for amplitude modulation overestimation. Moreover, it should be noted that, differently form the classical Taylor's hypothesis, the structure of $u(x)$ is different than the structure of $u(t)$ for the MTH case, since $U_{c}(t)$ is not a constant thus leading to a non-uniform spacing of the spatial series.

The $K_{n p}$ behaviour for the MTH is shown in cyan in Figure 5(b): the overestimation of $K_{n p}$ is compensated and its values are much more comparable to spatial-series obtained from DNS of the channel flow at $R e_{\tau} \approx 5200$ (black line in Figure 5(b)). This analysis confirms the applicability of the time-dependent correction of Yang \& Howland (2018) for amplitude modulation when time-series are employed, and extends such correction to the study of frequency modulation through (4.1). In particular, we stress that $u_{L S}(t)$ represents a more suitable choice than $u(t)$ in equation (4.1) since it is assumed that fluctuations in the large scale velocity, $u_{L S}(t)$, drive the variations in the friction velocity affecting the behaviour of small scales (see relation (4.2) and accompanying discussion). In this regard, the relation (4.1) leads to scaling arguments that are in good agreement with the quasi-steady quasi-homogeneous theory, as it will be discussed in $\S 4.2$.

One additional feature emerging from Figure 5 concerns the reversal in the modulation mechanism from the wall proximity to the outer flow. In fact, while Figure 5 shows a continuous decrease of $K_{n p}$ for $y^{+}>10$ and $y / h \lesssim 0.5$, an almost absence of FM was previously found in the log-region by means of other techniques (where a reversal of the FM was only detected in the proximity of the end of the boundary layer) (Ganapathisubramani et al. 2012; 
Baars et al. 2015). We point out that the $K_{n p}$ behaviour in Figure 5 resembles the decreasing behaviour of the AM parameters (e.g., see Mathis et al. 2009a,b), with a reversal of $K_{n p}$ from the near-wall (where $K_{n p}>1$ ) towards the outer region (where $K_{n p}<1$ ). In principle, if the small scales are both amplitude- and frequency-modulated, one could expect that following the Newtonian principle that to the same natural effects we must, as far as possible, assign the same causes - a similar underlying mechanism is at play for both amplitude and frequency modulation. This can justify the similarity between the $K_{n p}\left(y^{+}\right)$behaviour - that quantifies FM - with the widely reported behaviour of AM parameters, either in internal or external wall-bounded turbulent flows. In other words, both AM and FM result from a common underlying phenomenon, for which both amplitude and frequency of small scales are concurrently affected by negative or positive large-scale fluctuations at different wallnormal locations.

Specifically, concerning the reversal coordinate (i.e., the $y^{+}$-location where $K_{n p}$ switches from $K_{n p}>1$ to $K_{n p}<1$ ), for amplitude modulation the reversal typically occurs in the middle of the log-region, $y^{+} \approx 3.9 R e_{\tau}^{1 / 2}$ (Mathis et al. 2009a,b; Ganapathisubramani et al. 2012; Baars et al. 2015). For frequency modulation, instead, it is still not clear whether a modulation reversal does occur in the log-region (as shown in Figure 5) or it is only limited to the wake region (Baars et al. 2015). From Figure 5 we can conclude that FM mechanism is indeed limited to a near-wall region up to approximatively $y^{+}=100$, consistently with the analysis by Ganapathisubramani et al. (2012). Nevertheless, Figure 5 also shows that the reversal $y^{+}$location increases with the Reynolds number. In fact, we find that - when spatial-series are focused both from channel DNSs and time-series by the MTH - the reversal coordinates (i.e., $y^{+} \approx 35,75,145$ for the channel and boundary layer flows at $R e_{\tau}=1000,5186,14750$, respectively) scale as $y^{+} \approx 1.15 R e_{\tau}^{1 / 2}$ (the power-law fit gives an exponent of 0.5 with an $R^{2}=0.985$ ). This scaling has the same functional relationship found for amplitude modulation, i.e., $y^{+} \approx 3.9 R e_{\tau}^{1 / 2}$, but with a different proportionality constant. In particular, the $R e_{\tau}^{1 / 2}$-trend is reminiscent of the scaling of the outer peak position as a function of Reynolds number (Mathis et al. 2009a), thus strengthening the underlying connection of FM with the change in large scale features.

Another notable aspect discernible in Figure 5 is the V-like shape of $K_{n p}$ in the outer region of the channel flow, i.e. around $y / h \approx 0.5\left(y^{+} \approx 2500\right)$. In literature, the increase - giving the V-like shape - of amplitude modulation (AM) of small scales (which are representative of fine-scale turbulent motion) can be observed close to the channel center, e.g., in Chung \& McKeon (2010) (see Figure 4 therein) for the streamwise velocity, $u$, or in Yao et al. (2018) (see Figure 3c therein) for the $v$ and $w$ components. However, to the best of our knowledge, this peculiar increase of the modulation parameter in the channel flow has not been explicitly discussed so far. Here we propose an interpretation based on the insights gained from turbulent boundary layers.

Previous analyses, in fact, have highlighted that the preferential arrangement of the small scales in the wake region of turbulent boundary layers is mainly affected by intermittency, namely the presence of bulges of turbulent and non-turbulent flow (Baars et al. 2015, 2017). However, Figure 5(a) shows a similar V-like behaviour - as for previous results on AM also in the outer region of the channel flows, despite the absence of a turbulent/non-turbulent region in the channel flow (that is an internal flow). In particular, the V-like shape for the channel at $\operatorname{Re}_{\tau}=1000$ - although less evident than at $\operatorname{Re}_{\tau} \approx 5200$ - consistently occurs at $y / h \approx 0.5$ as highlighted in the inset of Figure 5(a). Here we suggest that - similarly to the effect of intermittency in boundary layer flows - the preferential arrangement in the proximity of the channel centreline could be affected by an alternating occurrence of highand low-rotational fluid motion above the head of large or very-large scale structures. This 
phenomenon would lead to the increase of both the AM and FM parameters toward the channel centreline. Although the clarification of this issue goes beyond the aim of this work, we do believe it deserves future investigations, being the channel flow setup much less considered for scale interaction analyses than turbulent boundary layers (for which high $R e_{\tau}$ data are much more available from experimental measurements).

With the aim to ensure that the behaviour of $K_{n p}\left(y^{+}\right)$described so far is the result of an intrinsic flow phenomenon rather than an artefact due to the network representation, in Figure 5 we also show the results for random-phase signals. Through a randomization of the phase of velocity Fourier coefficients, the energy spectral density and the turbulence intensity do not change, but any phase information is lost. Hence, following Mathis et al. (2009a), first the signals of $u$ are phase-scrambled, then the large scale component, $u_{L S}$, is extracted from the new random-phase signal (the amplitude spectrum is not changed), and eventually the degree is calculated from the full random-phase signal and conditioned to the sign of the random-phase $u_{L S}$. Figure 5 shows that, both in the channel flow and boundary layer setups, the characteristic behaviour of $K_{n p}$ for turbulent signals (black curves) disappears for randomphase velocity signals (blue curves). As previously reported (e.g., see Chung \& McKeon 2010), phase relationships between large and small scales play an important role in the characterization of scale interaction, specifically on scale modulation; thus, if any realistic phase information is lost, modulation effects disappear as well.

The NVG-based approach demonstrates to be reliable in capturing FM in turbulent velocity signals, and sensitive to phase-randomization. Moreover, the behaviour of $K_{n p}\left(y^{+}\right)$for both the channel and boundary layer is found to be robust under different cut-off wavelengths (used to extract $u_{L S}$ from the full signal $u$ ), as discussed in Appendix B. Further insights into the frequency modulation of the streamwise velocity will be presented in the next Section 4.2 focusing on the near-wall region, i.e., where large-to-small scale modulation does essentially take place.

\subsection{Scale interaction in the near-wall region}

The presence of a near-wall modulation, whose intensity increases with the Reynolds number, has posed a challenge to the classical view on the universality of near-wall turbulence, i.e., the independence of the near-wall statistics (scaled in wall units) to the Reynolds number when this is sufficiently large. Since large scale structures affect the behaviour of the wall shear stress (Mathis et al. 2013), the classical universality hypothesis has been recently replaced with the hypothesis that statistics have to be normalized by considering the large scale skin friction, $\tau_{L S}$, rather than the mean skin friction, $\tau_{w}$ (Zhang \& Chernyshenko 2016; Chernyshenko 2020). This hypothesis is referred to as quasi-steady quasi-homogeneous (QSQH) hypothesis, since the temporal and spatial variations of the large scale structures are much slower than variations of the near-wall turbulence (Zhang \& Chernyshenko 2016).

The aim of this section is (i) to provide the proportionality relationships between $u_{L S}$ and the (temporal or spatial) frequency of the small scales as expected from the QSQH hypothesis, and (ii) to test the validity of such relationships by means of the network degree centrality. In particular, we will focus on velocity signals extracted at $y^{+}=15$ that corresponds to the $y^{+}$value of maximum $K_{n p}$ in Figure 5, thus being a representative wall-normal coordinate of the near-wall region. This choice is also related to the fact that, how evidenced by Zhang \& Chernyshenko (2016) and Agostini \& Leschziner (2019), the validity of the QSQH hypothesis is found to be rather accurate only in a narrow region close to the wall that is $y^{+}<70-80$.

According to the QSQH hypothesis, variations in the large scale velocity, $u_{L S}$, induce proportional variations in the large scale skin friction, $\tau_{L S}$, namely $\left(\tau_{w}+\tau_{L S}\right) \propto\left(U+u_{L S}\right)$, 
where $U$ is the local mean velocity. Since, by definition, $U_{\tau}=\sqrt{\tau_{w} / \rho}$ (where $\rho$ is the fluid density), the effect of $u_{L S}$ on $\tau_{L S}$ can be stated in terms of velocities as

$$
\left(U_{\tau}+u_{\tau, L S}\right) \propto \sqrt{\left(\tau_{w}+\tau_{L S}\right)} \propto \sqrt{\left(U+u_{L S}\right)},
$$

where $u_{\tau, L S}$ is the fluctuating (i.e., zero-mean) large scale component of the friction velocity (Baars et al. 2017). Due to near-wall modulation, a quasi-steady or quasi-homogeneous variation of the friction velocity affects also the (amplitude and) frequency of the small scales. Specifically, $u_{L S}>0$ events induce $u_{\tau, L S}>0$ (see relation (4.2)) that, in turn, produces an increase of the small scale instantaneous spatial or temporal frequency according to the FM mechanism; vice versa for $u_{L S}<0$ (Baars et al. 2017).

In the near-wall region, the spatial scales are supposed to have a constant characteristic length when normalized in wall-units (e.g., see the inner spectral peak for $\lambda_{x}^{+}=\lambda_{x} U_{\tau} / v=$ const. $\approx 1000$ in Figure $3(\mathrm{a}))$. Therefore, spatial scales are related to $u_{\tau, L S}$ variations as $\lambda_{x}\left(U_{\tau}+u_{\tau, L S}\right)=$ const., namely $\lambda_{x} \propto 1 /\left(U_{\tau}+u_{\tau, L S}\right)$. Since spatial frequency (i.e., wavenumber), $\kappa_{x}$, is related to spatial scales as $\kappa_{x} \propto \lambda_{x}^{-1}$, by using the (4.2) we obtain the following scaling relations

$$
\lambda_{x} \propto\left(U+u_{L S}\right)^{-1 / 2}, \quad \kappa_{x} \propto\left(U+u_{L S}\right)^{1 / 2} .
$$

In the case of time-series, the temporal frequency, $f$, is related to spatial scales as $f=U_{c} / \lambda_{x}$, where $U_{c}$ is the convection velocity. Assuming that $U_{c}$ scales in wall units (Baars et al. 2017), namely $U_{c} \propto\left(U_{\tau}+u_{\tau, L S}\right)$, temporal frequency is eventually expected to scale as

$$
f \propto\left(U_{\tau}+u_{\tau, L S}\right)^{2} \propto\left(U+u_{L S}\right)
$$

Therefore, the relations (4.3) and (4.4) represent the expected scaling of spatial and temporal frequency, respectively, according the QSQH hypothesis.

As discussed in Section 2.3, the degree centrality represents a measure of the instantaneous wavelength or a temporal period. Therefore, it is expected that the degree, $k$, scales as $k \propto \lambda_{x}$ for the channel flow (in which spatial-series are mapped into NVGs), and $k \propto 1 / f$ for the boundary layer (in which time-series are analysed). If the degree is indeed an effective parameter to quantify FM, $k$ should then be proportional to $\left(U+u_{L S}\right)^{\beta_{u, x}}$ in the channel flow and $\left(U+u_{L S}\right)^{\beta_{u, f}}$ in the boundary layer. Following the aforementioned scaling arguments (i.e., relations (4.3) and (4.4)), the two exponents that verify the QSQH hypothesis should be equal to $\beta_{u, x}=-0.5$ and $\beta_{u, f}=-1$.

To test the $\beta_{u, x}$ and $\beta_{u, f}$ scaling, we conditionally averaged the degree centrality values (computed from NVGs of the full velocity signals $u(x)$ ) to the $u_{L S}$ values at $y^{+} \approx 10$. In particular, $u_{L S}$ values were firstly divided into uniformly-binned intervals in the range $\min \left[u_{L S}\right]-\max \left[u_{L S}\right]$. Then, for each visibility network (i.e., each signal), nodes $i$ for which $u_{L S}(i)$ belongs to a specific bin were selected, and the corresponding degree values, $k_{i}$, were averaged for that specific bin. By extending the averages to all $u_{L S}$ bins, the conditional average, $\left(\bar{k} \mid u_{L S}\right)$, is obtained, where the overbar indicates an average over a set of nodes. When plotting $\left(\bar{k} \mid u_{L S}\right)$, the $u_{L S}$ value representative of each bin is chosen as the middle value of the bin.

The behaviour of $\left(\bar{k} \mid u_{L S}\right)$ as a function of $u_{L S}$ reveals the scaling between degree-based frequency variations and large scale velocity variations. Such conditional degree averages are shown in Figure 6 for the channel flow at $R e_{\tau} \approx 5200$ (Figure 6(a)) and the boundary layer (Figure 6(b)), as a function of $\left(u_{L S} / U+1\right)$, where $U=U\left(y^{+}=10\right)$ is constant and $\left(u_{L S} / U+1\right)$ values equal to 1 correspond to large scale zero-crossing points $\left(u_{L S}=0\right)$. We find a scaling of the conditioned degree which follows a power-law with best-fit exponent $\beta_{u, x}=-0.48$ for the channel flow (spatial data) and $\beta_{u, f}=-1$ for the boundary layer when 
(a)

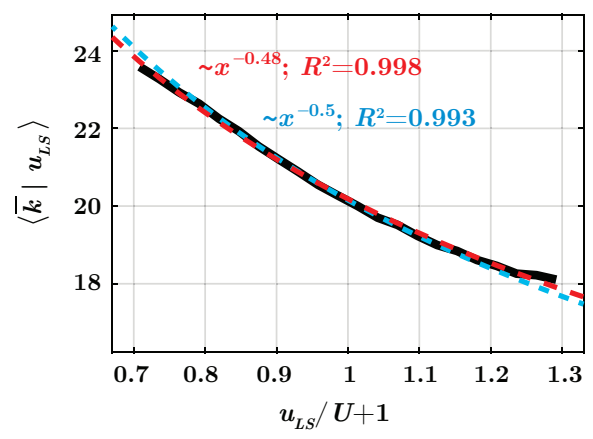

(b)



Figure 6: Average degree conditioned to the $u_{L S}$ values as a function of the normalized $u_{L S}$ deviation, for $u$ signals at $y^{+} \approx 10$ in (a) the turbulent channel flow and (b) the turbulent boundary layer (angular brackets indicate the average over time and homogeneous directions). In panel (b), the scaling for spatial-series obtained through the classical (CTH) and modified (MTH) Taylor's hypothesis are shown as black lines. The power-law fitting curves are shown as dashed lines, together with the exponent of the fitting and the coefficient of determination, $R^{2}$, for both setups. Light-blue dashed lines correspond to the expected scaling trends for spatial data. The intervals of $u_{L S} / U+1$ in abscissa cover, for each setup, a range from the 5 th to the 95th percentile of all $u_{L S}$ at the selected vertical coordinate $y^{+} \approx 10$.

local mean velocity is used in the Taylor's hypothesis (CTH). These exponent values are in excellent agreement with the expected values of -0.5 and -1 . We recall that, since for the $\mathrm{CTH}$ case the convection velocity is constant and equal to the local mean velocity $U$, the scaling exponent obtained in the CTH case is representative of a (temporal) frequency, thus obtaining $\beta_{u, f}=-1$. On the other hand, when the modified Taylor's hypothesis (MTH) is employed (equation (4.1)), the structure of the spatial-series (obtained from the corresponding time-series) significantly changes and scaling arguments are therefore congruent with DNS spatial-series. Accordingly, the $\left(\bar{k} \mid u_{L S}\right)$ scaling for the MTH case in Figure 6(b) produces an exponent which is close to -0.5 , as expected from spatial-series. Finally, we mention that an exponent $\beta_{u, x}=-0.5$ is found for the channel flow at $R e_{\tau}=1000$ with an $R^{2} \approx 0.92$ when cut-off filter is set to $\lambda_{x, c}^{+}=2500$, while for larger $\lambda_{x, c}^{+}$values a poorer fitting is observed, likely due to the limited scale separation for this setup.

While conditional averages were performed here by using uniformly-binned intervals of $u_{L S}$, Baars et al. (2017) - by adopting a variable-interval scheme for conditional averages reported a scaling of approximatively 0.8 (instead of 1 ) for frequency in turbulent boundary layers over a wide range of $R e_{\tau}$. They indicated that the discrepancy in the expected exponent might be caused by an inaccurate assumption that small scales are convected at a fixed innerscaled velocity. However, here we show that the expected scaling for $f$ is still obtained by assuming $U_{c} \propto\left(U_{\tau}+u_{\tau, L S}\right)$, suggesting that the discrepancy in the fitting in Baars et al. (2017) might be related to different methodological arguments.

The relevance of the scaling shown in Figure 6 is twofold. From one hand, it demonstrates that - similarly to the near-wall AM (Baars et al. 2017) - the near-wall FM agrees with the quasi-steady quasi-homogeneous hypothesis. On the other hand, the outcomes of Figure 6 further validate the capability of the visibility-based approach - relying on the degree centrality - to capture FM in wall-bounded turbulence, as well as the validity of the modified Taylor's hypothesis (4.1) in converting time-series into spatial-series. 
(a)

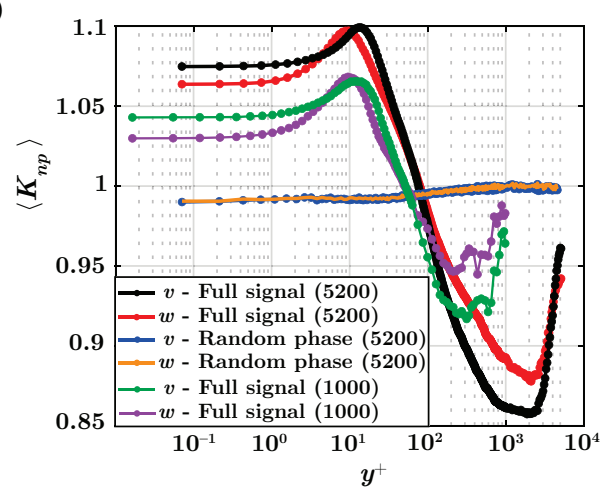

(b)



Figure 7: (a) $K_{n p}$ ratio as a function $y^{+}$for the wall-normal and spanwise velocity components, $v$ and $w$, extracted from the two channel flow DNSs, together with the corresponding $K_{n p}$ values for random-phase signals. The respective Reynolds number value of the DNS is reported within brackets in the legend. Angular brackets indicate averaging over time and spanwise direction. (b) Average degree conditioned to the $u_{L S}$ values as a function of the normalized $u_{L S}$ deviation, for $v$ and $w$ signals at $y^{+} \approx 10$ in the turbulent channel flow at $R e_{\tau} \approx 5200$. The power-law fitting curves are shown as cyan and green dashed lines for the $v$ and $w$ cases, respectively, together with the exponents of the fitting and the coefficients of determination, $R^{2}$.

\subsection{Analysis of the spanwise and wall-normal velocity components}

The application of the NVG approach to spatial-series of wall-normal, $v(x)$, and spanwise, $w(x)$, velocity from the DNS of the turbulent channel flows is here reported. Figure 7(a) shows the ratio $K_{n p}$ for the $v$ (black and green curves) and $w$ (red and purple curves) components as a function of $y^{+}$for the two channel flows at different $R e_{\tau} . K_{n p}>1$ is found in the near-wall region indicating a positive frequency modulation of the large scales on the small scales of $v$ and $w$. This result is consistent with amplitude modulation investigations, which show a similar modulating effect of the large scale motion on the small scales of the three velocity components (Hutchins \& Marusic 2007b; Talluru et al. 2014; Yao et al. 2018; Wu et al. 2019). In particular, the trends shown in Figure 7(a) are similar to that reported in Figure 5(a) for the $u$ signals, although lower $K_{n p}$ values are obtained from $v$ and $w$. Moreover, as for $u$, the FM of small scales of $v$ and $w$ is weaker at lower Reynolds number, since smaller $K_{n p}$ values are observed in the near-wall region for $\operatorname{Re}_{\tau}=1000$.

Furthermore, similarly to the $u$ component, an almost constant $K_{n p} \approx 1$ behaviour is found for network built from the random-phase $v$ and $w$ signals (see orange and blue curves in Figure 7 (a) referring to $R e_{\tau} \approx 5200$ as a representative case), confirming the ability of the degree to capture phase information from the (full) signal. It should be noted that, for the sake of consistency, a unique phase shuffling was performed in this case for the three velocity components. As the degree from the $v$ and $w$ signals are conditionally averaged on $u_{L S}$, the phase of the streamwise velocity signal was extracted and randomly shuffled, so that the random-phase $u_{L S}, v$ and $w$ signals were obtained via the respective (non-shuffled) amplitudes but with the same random phases.

Although spectral peak separation in the spectrograms of the transversal velocity components is less evident than for the streamwise velocity, the generation and amplification of small scale motions (i.e., fine scale vortices) of all the three velocity components is strongly connected with large scale events (Hutchins \& Marusic 2007b). Within this perspective, the wall-normal and spanwise velocities are expected to be modulated in the near-wall region by following the QSQH hypothesis in a similar way as the streamwise component, $u$. However, while results for AM of the three velocity components (Talluru et al. 2014; 
Agostini \& Leschziner 2019; Chernyshenko 2020) and scaling arguments for the AM and FM of the $u$ component (Baars et al. 2017) have been provided, as far as we know, similar scaling arguments (as in Baars et al. (2017), figure 9 therein) for $v$ and $w$ have not been pursued for FM to date.

In analogy with the modulation of the $u$ component, the conditional average degree, $\left(\bar{k} \mid u_{L S}\right)$, is evaluated as a function of $\left(u_{L S} / U+1\right)$ at $y^{+} \approx 10$ for NVGs built from $v(x)$ and $w(x)$ signals. The conditional average degree and the corresponding fitting are shown in Figure 7(b) for the $R e_{\tau} \approx 5200$ setup and confirm the power-law modulation effect of the large scales in the near-wall region even for the other velocity components, namely $k \propto\left(U+u_{L S}\right)^{\beta_{v, x}}$ and $k \propto\left(U+u_{L S}\right)^{\beta_{w, x}}$. However, while for the $u$ component the exponent of the power-law was $\beta_{u, x} \approx-0.5$, a weaker scale interaction effect is found for the $v$ and $w$ components being $\beta_{v, x} \approx-0.3$ and $\beta_{w, x} \approx-0.3$, which are both smaller (in modulus) than $\beta_{u, x}$. This outcome is also consistent with the smaller $K_{n p}$ values for $v$ and $w$ (see Figure 7(a)) than for $u$ (see Figure 5(a)), indicating a weaker FM in the near-wall region for the transversal velocity components.

The power-law relationships found for $u, v$ and $w$ suggest that - although the intensity of modulation is different for each velocity component - the response of the small-scales of $v$ and $w$ exhibits a functional relation qualitatively analogous to the response of $u$. In general, there could be several factors playing a role in the scale-interaction mechanisms (such as the direction of the large-scale motions as discussed by Chernyshenko (2020)), but we can conclude that the QSQH hypothesis is valid for all velocity components, although a more refined description is required for the transversal components, $v$ and $w$.

The results shown in this section reveal that the three velocity components are all affected by a large scale FM in the near-wall region, where an increase of the local (spatial) frequency is observed under $u_{\tau, L S}>0$ periods induced by positive $u_{L S}$ events. In particular, we provided novel insights on FM for the $v$ and $w$ components - which have been investigated less than $u$-in terms of FM intensity for spatial-series (Figure 7(a)), and scaling arguments on the QSQH hypothesis (Figure 7(b)).

\subsection{Time and space shifting in FM}

To conclude our analysis, we provide results on the investigation of time- and space-shifted FM, as quantified by $K_{n p}$. We recall that a lead of the small scale amplitude was found with respect to the large scales in the near-wall region of turbulent boundary layers, while a small scale lag is found above the reversal coordinate (Bandyopadhyay \& Hussain 1984; Guala et al. 2011). Concerning FM, a lead of the small scale frequency with respect to large scales was found in the near-wall region but, differently from AM, scattered behaviours were found far from the wall (Ganapathisubramani et al. 2012; Baars et al. 2015).

To address this issue, we show in Figure 8 the conditionally average degree, $K_{n p}$, as a function of $y^{+}$and the spatial delay, $\Delta r_{x}^{+}$, where for time-series it holds the Taylor's hypothesis $\Delta r_{x}^{+}=-U_{c} \Delta t^{+}$(the minus sign highlights the opposite direction of reference systems between fixed-point time-series and spatial-series). Therefore, the formulation of $K_{n p}$ reported in (2.3) is extended to account for spatial shifting, $\Delta r_{x}^{+}$, as $K_{n p}\left(\Delta r_{x}\right)=K_{n}\left(\Delta r_{x}\right) / K_{p}\left(\Delta r_{x}\right)$, with

$$
\left\{\begin{array}{l}
K_{n}\left(\Delta r_{x}\right)=\frac{1}{N_{n e g}\left(\Delta r_{x}\right)} \sum_{j=1}^{N}\left(k\left(x_{j}-\Delta r_{x}\right) \mid u_{L S}\left(x_{j}\right)<0\right), \\
K_{p}\left(\Delta r_{x}\right)=\frac{1}{N_{\text {pos }}\left(\Delta r_{x}\right)} \sum_{j=1}^{N}\left(k\left(x_{j}-\Delta r_{x}\right) \mid u_{L S}\left(x_{j}\right)>0\right) .
\end{array}\right.
$$

Positive or negative $\Delta r_{x}^{+}$values indicate in (4.5) a lag or lead, respectively, of the degree, $k$, with respect to $u_{L S}$ in the conditional averages of equation (2.3) (the results in Figure 5 correspond to $\Delta r_{x}^{+}=\Delta t^{+}=0$ ). If classical Taylor's hypothesis $(\mathrm{CTH})$ is employed for 
(a)



(b)

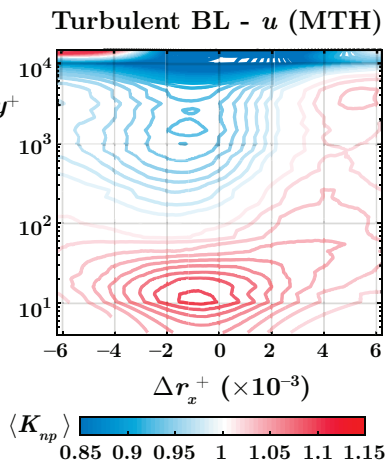

(c) Turbulent channel flow - $u$

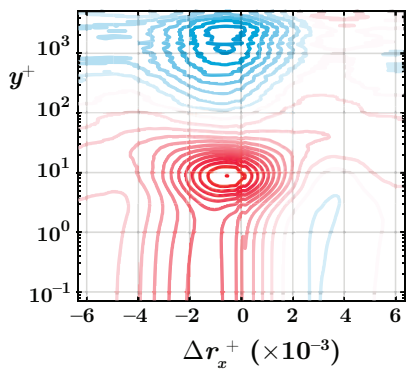

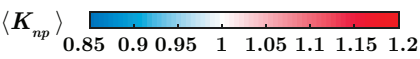

(d)

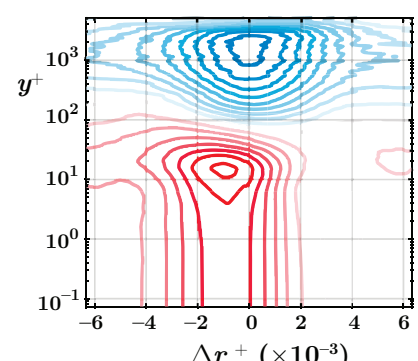

$\Delta r_{x}^{+}\left(\times 10^{-3}\right)$



(e)

Turbulent channel flow - $w$

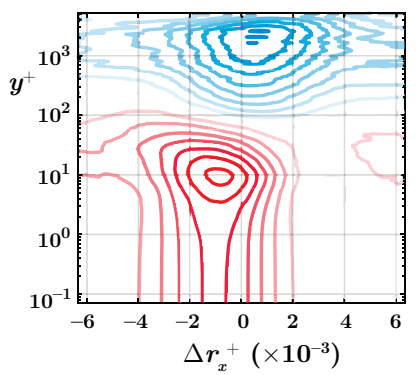

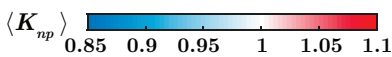

Figure 8: Contour plot of the $K_{n p}$ ratio as a function of the wall-normal coordinate, $y^{+}$, and the temporal or spatial shifting, $\Delta t^{+}$or $\Delta r_{x}^{+}$, respectively. Shifting for the turbulent boundary layer are reported in panels (a) and (b) whether the classical or modified Taylor's hypothesis is used, respectively. Spatial shifting for the turbulent channel flow at $R e_{\tau} \approx 5200$ is shown in panels (c-e) for the three velocity components. Iso-level contours are displayed by using a level-step equal to 0.03 in panel (a), 0.01 in panel (b) and 0.015 in panels (c)-(e).

turbulent boundary layer time-series (Figure 8(a)), a slight lead of $K_{n p}$ with respect to $u_{L S}$ (i.e., high $K_{n p}$ values are at $\Delta r_{x}^{+}<0$ but close to $\Delta r_{x}^{+}=0$ ) is observed for $y^{+} \lesssim 15$. However, a more substantial lead is observed for larger $y^{+}$coordinates in the near-wall region up to $y^{+} \approx 100$, in agreement with previous analyses (Baars et al. 2015), while a lag of $K_{n p}$ with respect to large scales is detected for $y^{+} \gtrsim 100$. A clearer picture is obtained when the modified Taylor's hypothesis (MTH) is exploited (Figure 8(b)). Significant lead of $K_{n p}$ with respect to $u_{L S}$ is found in the whole near-wall region (including wall proximity, $y^{+} \lesssim 15$ ), while the lag for $y^{+} z 100$ is less evident and a lead is recovered for larger $y^{+}$values (see blue contours in Figure 8(b)). Eventually, no clear patterns are observed in the intermittent regions $\left(y^{+} \geq 5 \times 10^{3}\right)$.

The space-shifted $K_{n p}$ values in the turbulent channel flow at $\operatorname{Re}_{\tau} \approx 5200$ for the three velocity components are displayed in Figure $8(\mathrm{c}-\mathrm{e})$. Likewise the turbulent boundary layer, a lead of $K_{n p}$ with respect to $u_{L S}$ is found for $y^{+}<100$, as highlighted by high $K_{n p}$ values for $\Delta r_{x}^{+}<0$. Differences among the $u, v$ and $w$ components are detected in proximity of the channel centreline, where $K_{n p}$ appears to lead, be in-phase, and slightly lag $u_{L S}$ for the $u$ (Figure 8(c)), $v$ (Figure 8(d)), and $w$ component (Figure 8(e)), respectively. It should be noted that the $R e_{\tau} \approx 5200$ is here used as a representative setup for spatial data, and results for the lower $R e_{\tau}$ channel flow are in agreement with results in Figure 8 so they are not shown 
for the sake of conciseness.

In particular, it is worth highlighting that the maximum $K_{n p}$ values in the near-wall region are found at $\Delta r_{x}^{+} \approx 1000$ for the streamwise velocity in the turbulent boundary layer when the MTH is employed (Figure 8(b)), as well as for all the velocity components in the turbulent channel flow (Figure $8(\mathrm{c}-\mathrm{e})$ ). The value $\Delta r_{x}^{+} \approx 1000$ is in very good agreement with the characteristic length scale in the near-wall region, being $\lambda_{x}^{+}=O\left(10^{3}\right)$ (see inner spectral peak in Figure 3(a)). The equivalent time-scale is $\Delta t^{+}=\Delta r_{x}^{+} / U_{c}^{+} \approx 100$ (being $U_{c}^{+} \approx 10$ in the buffer layer and viscous sublayer), which is the characteristic turnover time of the near-wall cycle. As small scales are supposed to be actively modulated by large-scales, the time taken for this process to be completed is therefore equivalent to the time scale of the near-wall cycle (Ganapathisubramani et al. 2012).

The results shown in Figure $8(\mathrm{a}-\mathrm{c})$ for the streamwise velocity reveal that different convection velocities indeed play a significant role in the FM dynamics, not only in terms of overestimation (as highlighted in Figure 5(b)), but also in terms of spatial delay that in the near-wall region - is strongly related to the near-wall cycle. Therefore, NVG reveals again to be a reliable approach for quantifying FM even in presence of a temporal or spatial shifting. Finally, we note that an important issue about scale interaction is whether large scales actually cause an increase or decrease of small scale activity, as the parameters used so far to quantify AM and FM only show there is a relation (e.g., a correlation) between large scales and small scales. Although definite answers to this issue are not still available, our detection of the presence of a significant temporal or spatial delay close to the characteristic time or length scale of the near-wall cycle, in conjunction with the arguments leading to relation (4.2), could provide supporting clues that a causation process is at play. In fact, fluctuations in the large scale component of the wall shear stress - which affect the small scales behaviour - appear to be directly caused by the outer large scale structures rather than being the feature of near-wall processes (Zhang \& Chernyshenko 2016).

\section{Discussion}

In this work, the natural visibility graph was used to study 1D spatial-series and time-series from two turbulent flow configurations, but some generalizations can be carried out. First, the geometrical criterion at the basis of the visibility algorithm can be extended to scalar fields of arbitrary dimension (Lacasa \& Iacovacci 2017). For instance, Tokami et al. (2020) recently constructed a spatial visibility graph (employing a simplified version of the NVG called horizontal visibility graph as proposed by Luque et al. (2009)) from a 2D velocity field in a buoyancy-driven turbulent fire. Therefore, our approach could be extended to $2 \mathrm{D}$ velocity fields at fixed $y^{+}$coordinates, thus concurrently taking into account the degree variations along the streamwise and spanwise directions.

The results provided by natural visibility graphs, specifically about the degree centrality, necessarily depend on the signal resolution (either the sampling frequency or the grid size), which needs to be sufficiently high to capture the behaviour of small scales. However, if the temporal or spatial resolution is sufficient enough to capture the smallest significant features of the signal, the degree centrality tends to proportionally scale with the signal resolution as shown, e.g., in Iacobello et al. (2018b). Nevertheless, an additional feature of visibility networks is the possibility to explicitly account for the spatial or temporal discretization. In fact, one can assign to each discrete observation, $i$, the corresponding signal spacing (e.g., $\Delta x_{i}, \Delta z_{i}, \Delta t_{i}$, etc.). In this way, each node $i$ of the network is representative of an interval centred in $i$, thus providing a continuous representation of the signal. As a result, a weighted network is obtained in which the relation 2.2 is reformulated as $\tilde{k}_{i} \equiv \sum_{j} \Delta \chi_{j} A_{i, j}$, for a series sampling, $\Delta \chi_{j}$, where $\chi$ is independent variable (e.g., time). This generalization is 
particularly useful for non-uniformly sampled signals from experimental measurements, in which $\tilde{k}$ can be used in place of $k$, e.g., in the definition (2.3).

Finally, it is worth to observe that, so far, the visibility approach was presented as a convexity criterion (see inequality (2.1)). In particular, the network degree was interpreted as a measure of the instantaneous period (quantified in terms of the local convexity of the signal), in analogy with the concept of instantaneous frequency based on the Hilbert transform (where the local properties of a series are emphasized by performing a convolution of the signal with the function $1 / t$ (Huang et al. 1998)). Nevertheless, the visibility algorithm can also be used as concavity criterion by applying it to the opposite signal, $-s_{i}$, whose effect is to change the direction in the inequality (2.1) (Iacobello et al. 2019a). The comparison of the network metrics extracted from $s_{i}$ and $-s_{i}$ allows one to characterize the peak-pit asymmetry of a signal, especially in real-world phenomena (Hasson et al. 2018). Following this point of view, we evaluated - for the sake of completeness - the values of $K_{n p}\left(y^{+}\right)$by using the NVG as a concavity criterion for the streamwise velocity, and we found that the main features of the FM for full and random-phase signals are retained either when the information is only taken from the convexity or concavity criterion.

\section{Conclusions}

In this study, we propose a novel approach to investigate the frequency modulation (FM) mechanism in wall-bounded turbulence by means of the natural visibility graphs. Spatialseries and time-series of the velocity from two turbulent channel flows and a turbulent boundary layer, respectively, are mapped into visibility networks and the degree centrality is conditionally averaged to the sign of the large scale velocity to quantify FM. In particular, the versatility of visibility graphs to map either time- or spatial-series, let us exploit velocity spatial-fields from turbulent channel flows that have been much less investigated than turbulent boundary layers under the lens of frequency modulation.

The overall results for the streamwise velocity indicate a frequency modulation mechanism occurring in the near-wall region with a peak of intensity in the buffer layer, in agreement with previous works. However, in contrast with previous observations on FM, we observe a reversal in the frequency modulation mechanism far from the wall similarly to what observed for amplitude modulation, in both channel and boundary layer flows. We argued that such similarity could stem from a common underlying phenomenon, for which both amplitude and frequency of small scales are concurrently affected by negative or positive large-scale fluctuations. Moreover, we observe that the reversal coordinate scales as $R e_{\tau}{ }^{0.5}$, which is reminiscent of the scaling in the wall-normal position of the outer spectral peak.

The effect of different convection velocities for the time-series analysis is also discussed. In particular, we modified the correction proposed by Yang \& Howland (2018) by accounting for only the large scale velocity component in the definition of the convection velocity. This choice is based on the rationale that variations in the large scale velocity induce variations in the wall shear stress, which in turn affect the behaviour of small scales. We detect an overprediction of frequency modulation when the local mean velocity is employed as convection velocity in the turbulent boundary layer, while such overprediction is compensated when the proposed modified Taylor's hypothesis is used. Moreover, scaling behaviours of the degree centrality as a function of the large scale velocity are found to be in very good agreement with the the quasi-steady quasi-homogeneous (QSQH) theory. In this regard, our correction of the Taylor's hypothesis provides reliable scaling trends as the large scale velocity is supposed to induce modulation of small scales through variations in the wall shear stress.

Finally, the FM for the wall-normal and spanwise velocity components is analysed for 
the turbulent channel flows and FM scaling is discussed for the transversal velocities. We find a frequency modulation mechanism for the wall-normal and spanwise velocity components qualitatively similar to FM of the streamwise velocity. Specifically, a powerlaw scaling of the degree conditioned to the large scale velocity is found for the three velocity components, although smaller exponents are found for transversal velocities than for the streamwise velocity. Moreover, a delay-based analysis is carried out for the three velocity components in the channel flow and for streamwise velocity time-series in the boundary layer. We observe that small scales lead large scales in the near-wall region (in accordance with previous studies), but significant differences are found when the classical or modified Taylor's hypothesis is applied. Specifically, our modified Taylor's hypothesis provides results in agreement with spatial-series analysis, where the delay of maximum modulation corresponds to the characteristic length (or temporal) scale of the near-wall cycle.

Furthermore, we emphasize here that, to the best of our knowledge, this is the first time that frequency modulation is thoroughly investigated for all the three velocity components, as previous works have been mainly focused on amplitude modulation. The findings gained through the visibility networks of all the three velocities can then contribute to the development of a more general model of scale interaction, which accounts for the different modulating effect of the large scale on each velocity component.

The visibility-based approach reveals to be robust in the quantification of FM with respect to AM (Appendix A), and to different cut-off filtering sizes and high-frequency noise (Appendix B), as well as sensitive to a spectral phase randomization of the signals. The latter implies that the natural visibility graph is able to capture non-linearities in the signal, as linear effects are preserved during phase randomization (i.e., amplitude spectrum does not change) while non-linearities are lost through phase-shuffling. We stress that the visibility networks do not require any a priori parameter, and are directly built from the full velocity signals (instead of the small scale component), being the network degree able to capture the signal structure at local scales. In this regard, although in this work a one-point analysis is carried out for simplicity, a two-point analysis (where the large scale signal is extracted at a fixed wall-normal coordinate) would reveal the full potential of visibility networks. In fact, when multiple synchronized signals are available at different wall-normal locations (e.g., from numerical simulations, hot-wire rakes or through particle image velocimetry), the large scale signal can be obtained only once at a fixed wall-normal location, as well as probes working on a smaller frequency range can be employed (being only low-frequencies necessary). The full velocity signals, instead, can be used without any filtering operation to capture the small scales frequency modulation at the remaining wall-normal locations.

In the wake of the recent successful applications of network science to the analysis of turbulent flows (Murugesan \& Sujith 2015; Taira et al. 2016; Schlueter-Kuck \& Dabiri 2017; Krishnan et al. 2019; Iacobello et al. 2019b), the proposed visibility-based approach candidates for being a parameter-free and robust tool for FM investigation.

\section{Appendix A. Synthetic signals for visibility-based FM detection}

In this appendix we provide results of the application of the visibility-based approach to quantify frequency modulation from synthetic signals, which is a simple but representative benchmark of more complex signals such as from turbulent flow fields. Three configurations of modulation are here investigated, as shown in Figure 9(a-c), namely amplitude modulation $(\mathrm{AM})$, frequency modulation (FM) and both amplitude and frequency modulation (AFM). In this way, we assess the effect of different modulations on the ratio $K_{n p}$ and its ability to discern FM only. 
(a)

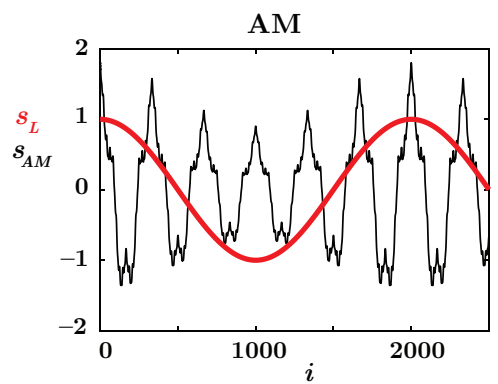

(c)

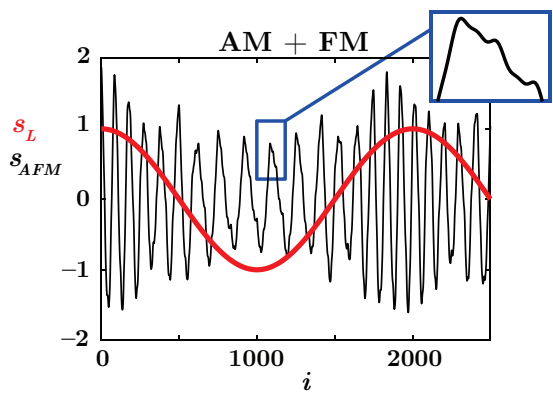

(b)

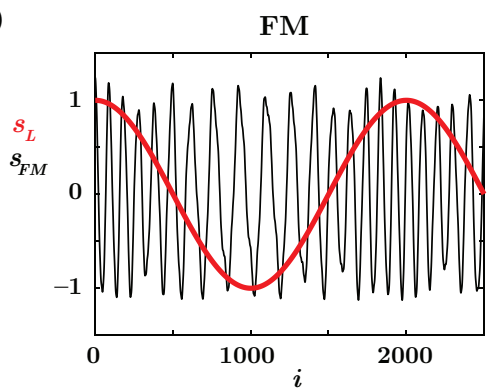

(d)

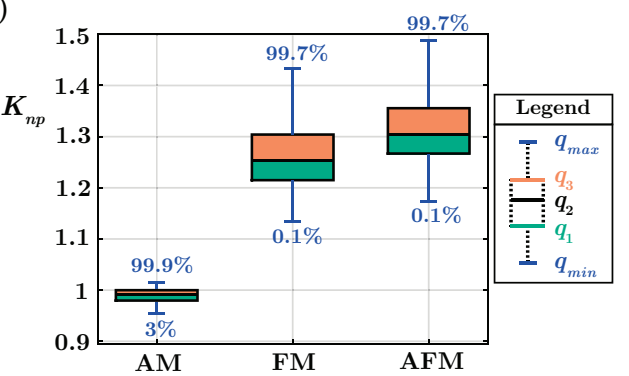

Figure 9: First 2500 time instants (out of $10^{4}$ ) of the three synthetic modulated signals (shown in black) and the modulating signal (shown in red) for: (a) amplitude modulated signal (AM); (b) frequency modulated signal (FM); (c) amplitude and frequency modulated signal (AFM). The inset show a zoom of the AFM modulated signal. (d) Results of the application of the NVG to the synthetic signals in panels (a-c). Values of $K_{n p}$ are shown as box plots, where $q_{1}, q_{2}$ and $q_{3}$ are the 25th, median, and 75 th percentiles, respectively, while $q_{\min }=q_{1}-1.5\left(q_{3}-q_{1}\right)$ and $q_{\max }=q_{1}+1.5\left(q_{3}-q_{1}\right)$ whose values are explicitly indicated at the tips of the whiskers as percentages.

All the generated signals have length $N=10^{4}$ and sampling frequency $f_{\text {samp }}=4000 \mathrm{~Hz}$, which is chosen to be much larger than the characteristic frequencies of the modulated and modulating signals. The modulating (i.e., large scale) signal - shown in red Figure 9(a-c) is given by the expression $s_{L}\left(t_{i}\right)=\cos \left(2 \pi f_{L} t_{i}\right) / 3$, where $t_{i}=(i-1) / f_{\text {samp }}$ is time, with $i=1, \ldots, N$, and $f_{L}=2 \mathrm{~Hz}$ is the frequency of the modulating signal.

The three modulated signals, $s_{A M}, s_{F M}$ and $s_{A F M}$, are constructed as high-frequency sinusoidal series, which emulate the small scale velocity component, modulated by $s_{L}$. A positive modulation is considered, namely, an increase of amplitude and/or frequency is induced for intervals of positive $s_{L}$ values, and vice versa for negative $s_{L}$ values. This behaviour mimics the modulation close to the wall by large scales to small scales in wall-bounded turbulence. Specifically, the three modulated signals, shown as black lines in Figure $9(\mathrm{a}-\mathrm{c})$, are given as follows:

- $s_{A M}=\left(\cos \left[2 \pi f_{H} t_{i}\right]+s_{R}\right)\left(1+s_{L}\right)$,

- $s_{F M}=\cos \left[2 \pi f_{H} t_{i}+\varphi_{L}\right]+s_{R}$,

- $s_{A F M}=s_{F M}\left(1+s_{L}\right)$,

where $f_{H}=12 \mathrm{~Hz}$ is the (high) carrier-frequency of the modulated signals. The role of $\left(1+s_{L}\right)$ is to provide the amplitude modulation effect on $s_{A M}$ and $s_{A F M}$, while the role of $\varphi_{L}$ is to give a frequency modulated component to $s_{F M}$ and $s_{A F M}$. In particular, $\varphi_{L}$ is a time-varying phase depending on $s_{L}$ typically used to generate frequency-modulated signals (Boashash 2015), defined as $\varphi_{L}=2 \pi f_{\Delta} I_{m}$, where $f_{\Delta}=11 \mathrm{~Hz}$ is the frequency deviation (i.e., the maximum frequency shift from $f_{H}$ ), and $I_{m} \equiv \sum_{i} s_{L}\left(t_{i}\right) / f_{\text {samp }}$. The frequency deviation, $f_{\Delta}$, is selected to be close to the value of the carrier frequency, $f_{H}$, in order to maximize the modulation effect on the signal. 
In each of the three modulated series, an additional signal, $s_{R}$, is also included. It is obtained as a sum of unmodulated cosine signals with randomly-varying amplitude, given by

$$
s_{R}\left(t_{i}\right)=\sum_{q=2}^{5} \frac{r_{A}}{2^{q}} \cos \left[2 \pi\left(2^{q} f_{H}\right) t_{i}\right],
$$

where $r_{A}$ is a random number extracted from a uniform distribution in the range $(0,1)$. The effect of $s_{R}$ in a modulated signal can be observed in the inset of Figure 9(c). The role of $s_{R}$ is to introduce - similarly to turbulence velocity spectra - additional high-frequency low-amplitude components, thus making the modulated (small scale) signal a broadband-like series.

By generating several random amplitudes, $r_{A}$, in equation $\mathrm{A} 1$, an ensemble of $s_{R}$ series is obtained for each $r_{A}$. This leads to an ensemble of different modulated signals, $s_{A M}, s_{F M}$ and $s_{A F M}$, that are characterized by different $s_{R}$. Specifically, we generated $5 \times 10^{3}$ values of $r_{A}$ for each of the three modulated signals. The values of the ratio $K_{n p}$ (see equation 2.3) are then computed for each ensemble, by evaluating the degree on the NVGs built for the full signals, namely, $\left(s_{A M}+s_{L}\right),\left(s_{F M}+s_{L}\right)$ and $\left(s_{A F M}+s_{L}\right)$.

Figure $9(\mathrm{~d})$ shows the values of $K_{n p}$ for the three modulation configurations as box-plots, in which the most significant percentiles are highlighted. For the AM case, $K_{n p}$ is concentrated around unity, with a median value that is very close to one, as expected since the main modulating effect is on amplitude. For the FM and AFM cases, instead, values of $K_{n p}$ greater than one are consistently obtained (note the percentile values in Figure 9(d)), as a result of the positive frequency modulating effect of the large scale signal. In particular, it is worth noting that even when a signal is modulated both in amplitude and frequency, the ratio $K_{n p}$ is able to emphasize the contribution of the FM.

The results shown in Figure 9(d) reveal that $K_{n p}$ is an accurate parameter to quantify FM, since it consistently shows positive values under positive FM, and also a precise metric, since there is narrow spreading of the $K_{n p}$ values around the median. The results shown in this appendix corroborate the ability and robustness of the proposed visibility-based approach relying on the conditioned degree centrality - to capture frequency modulation, thus fostering its application as a tool to study scale-interaction in wall-bounded turbulence.

To conclude this section, we show the effect of higher frequency harmonics on the average degree, $K$, for synthetic signals. With this aim, we used two sets of synthetic signals according to their power spectrum scaling: (i) following a $-5 / 3$ spectrum, and (ii) a -2 spectrum. The former emulates turbulent signals in the inertial range, while the second refers to signals defined in equation (A 1). Figure 10(a-b) show the power spectra for both types of synthetic signals, while Figure 10(c) illustrates the behaviour of $K$ as a function of the maximum frequency considered, $f^{*}$. As discussed in $\S 2.2$ referring to Figure 3(c) for turbulent series, $K$ decreases as the number of high-frequency harmonics increases. Moreover, the changes in $K$ are stronger for the synthetic signals following the $f^{-5 / 3}$ spectrum (Figure 10(a)) than for $f^{-2}$ spectrum (Figure 10(b)), because the energy content of small scales is larger in the former case being the exponent $-5 / 3$ lower (in modulus) than -2 .

\section{Appendix B. Sensitivity analysis}

The aim of this section is to assess the robustness of the proposed NVG-based approach under different values of the spectral filtering wavelength and under high-frequency noise.

We recall that the spectral filtering wavelength is used to extract the large scale component, $u_{L S}$, from the streamwise velocity signal, $u$. Mathis et al. (2009a) firstly reported a sensitivity 
(a)

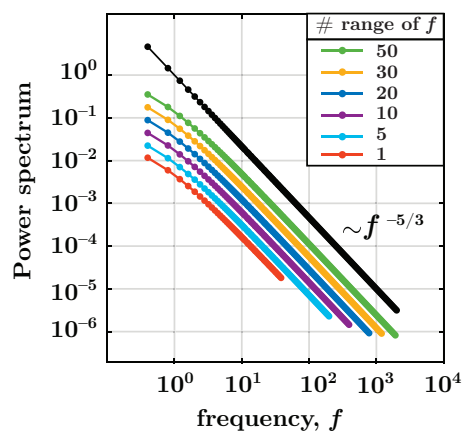

(b)

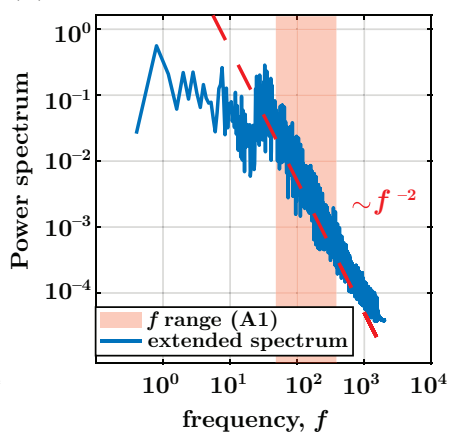

(c)

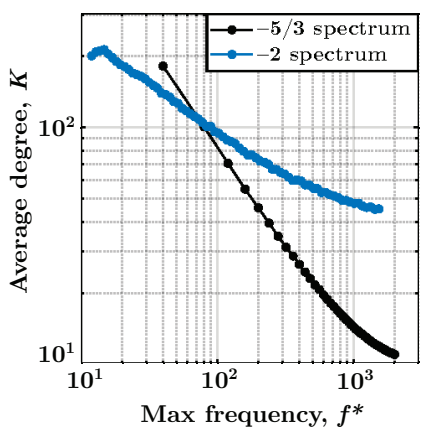

Figure 10: (a) Power spectra of synthetic signals following a $f^{-5 / 3}$ law. Signals are generated through inverseFourier transform of the power spectrum shown in black for different frequency ranges (i.e., different number of harmonics). Fifty ranges of $f$ are generated, and the spectra of the synthetic signals for some representative $f$ ranges are highlighted with different colours, as well as vertically shifted to enhance visualization. (b) Power spectrum of signals generated as per equation (A 1). The range of frequency considered for the equation (A 1 ) is highlighted as a shaded red region, and an $f^{-2}$ scaling is also shown. (c) Average degree centrality, $K$, for signals generated as in (a) and (b) for increasing maximum frequency, $f^{*}$.

analysis on the AM of streamwise velocity in a turbulent boundary layer. They showed that a decrease of the cut-off wavelength leads to a small increase of the AM below the reversal wall-normal coordinate (i.e., in the near-wall region), and a small decrease of AM above the reversal coordinate (i.e., far from the wall). The conclusion was that, despite the small variations due to different cut-off wavelengths, the general form of the AM parameter is retained. For this reason, subsequent works on AM and FM exploited the sensitivity analysis by Mathis et al. (2009a) as a reference case to justify the choice of the cut-off wavelength.

Here we perform a sensitivity analysis on the wall-normal behaviour of $K_{n p}$ for the streamwise velocity, by changing the cut-off wavelength, $\lambda_{x, c}$. Figure 11 shows $K_{n p}$ as a function of $y^{+}$for four $\lambda_{x, c}$ values, in the turbulent channel flow at $R e_{\tau} \approx 5200$ (Figure 11(a), $\lambda_{x, c}^{+}=5186$ in the main text) and the turbulent boundary layer (Figure 11(b), $\lambda_{x, c}^{+}=7000$ in the main text). For the boundary layer, both the classical and modified Taylor's hypotheses are considered and labelled as CTH and MTH in the legend of Figure 11(b). The nominal shape of $K_{n p}$ as a function of $y^{+}$is maintained both for the channel and boundary layers setups, and - similarly to the analysis carried out by Mathis et al. (2009a) - a decrease in $\lambda_{x, c}$ leads to a reduction of $K_{n p}$ below the reversal $y^{+}$and a rise of $K_{n p}$ above the reversal $y^{+}$. Specifically, variations of $K_{n p}$ in the wall proximity are less evident for the boundary layer when the modified Taylor's hypothesis (MTH) is applied rather than when local mean velocity is considered as convection velocity $(\mathrm{CTH})$.

This sensitivity analysis confirms the robustness of the decoupling procedure to extract $u_{L S}$, which is employed to evaluate $K_{n p}$ as metric for studying frequency modulation.

Finally, our method is tested under the presence high-frequency noise in the velocity signals (as usually happens in experimental measurements). With this aim, we artificially added a high-frequency noise to experimental signals of the streamwise velocity (whose sampling frequency is $f_{s}=20000 \mathrm{~Hz}$ ) in the turbulent boundary layer. The noise signal is given by the sum of three harmonics with random phase and with frequencies equal to $0.5 f_{s}, 0.475 f_{s}$ and $0.45 f_{s}$ (the maximum frequency included is $f_{s} / 2$ as higher frequencies are not be captured in the amplitude spectrum), whose effects on spectra are displayed in Figure 12(a). The corresponding values of $K_{n p}$ are shown in Figure 12(b), where we observe that the behaviour of $K_{n p}$ is retained throughout the boundary layer except for the intermittency region where 
(a)

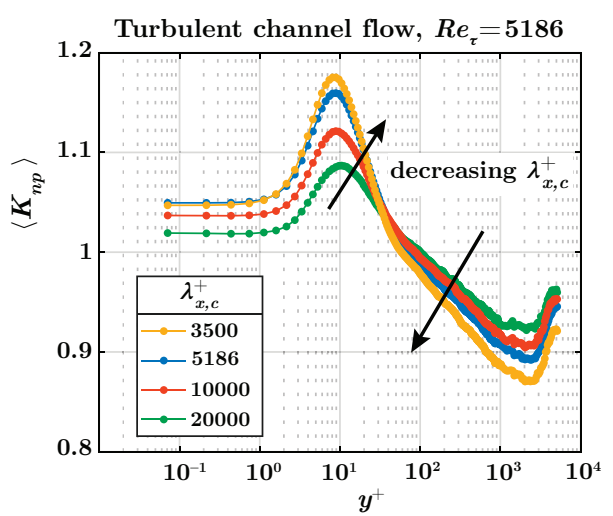

(b)

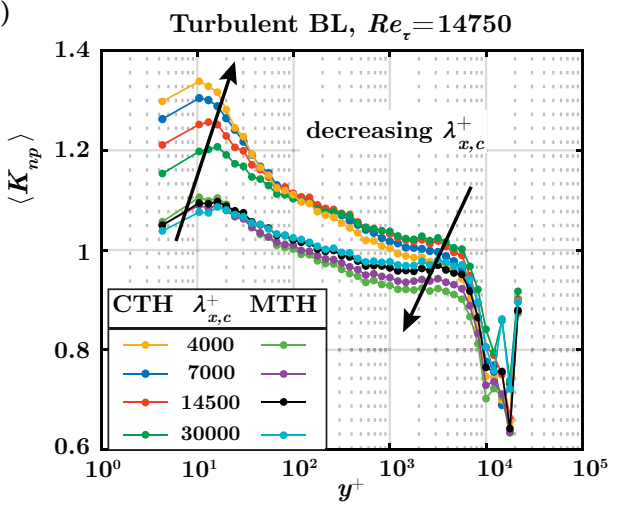

Figure 11: Effect of different cut-off wavelengths in the large scale conditional average degree ratio, $K_{n p}\left(y^{+}\right)$, for streamwise velocity, $u$, extracted from (a) the channel flow DNS at $R e_{\tau} \approx 5200$ and (b) the boundary layer experiments. In panel (b), the cut-off effect for spatial series obtained from time-series via classical Taylor's hypothesis $(\mathrm{CTH})$ - namely using as convection velocity the local mean velocity - and modified Taylor's hypothesis (MTH). Angular brackets indicate averaging over time and spanwise direction in (a) and over three different realizations in (b).
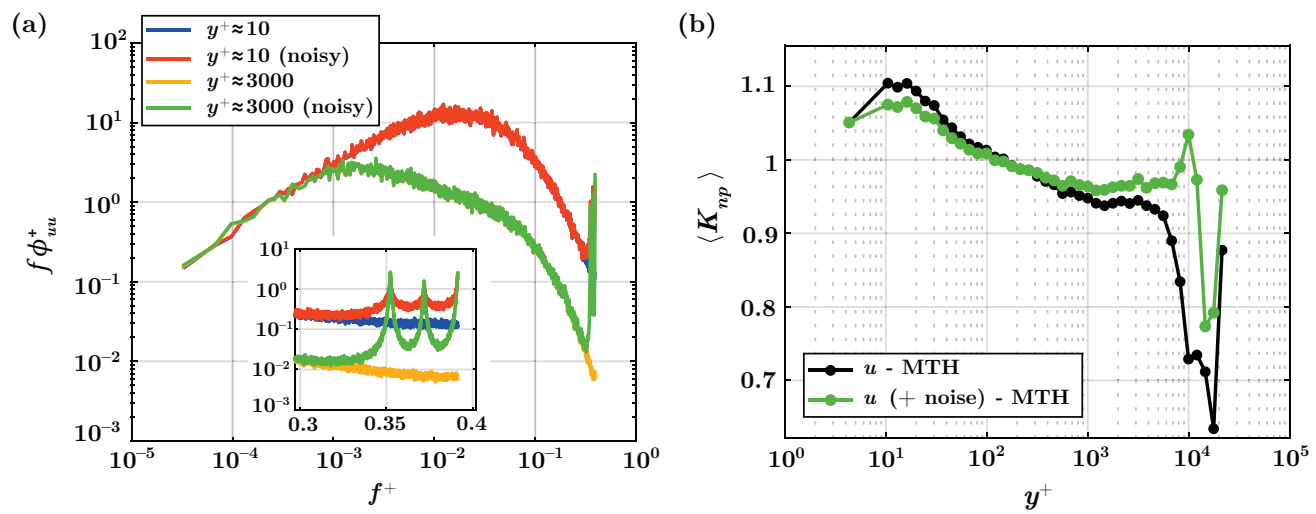

Figure 12: (a) Pre-multiplied energy spectrum of the streamwise velocity, $u u$, from the turbulent boundary layer at two representative $y^{+}$locations with and without high-frequency noise. The inset shows a zoom at the highest frequencies. (b) $K_{n p}$ as a function of $y^{+}$for velocity signals without (black) and with (green) high-frequency noise (the MTH is used).

the noise intensity significantly affects the signal structure. Therefore, we conclude that although a pre-processing of the (experimental) data is always a good practice to avoid biased behaviours - the NVG approach based on $K_{n p}$ is sufficiently robust under high-frequency noise.

Declaration of interests. The authors report no conflict of interest.

Author ORCID. G. Iacobello, https://orcid.org/0000-0002-0954-8545; L. Ridolfi, https://orcid.org/00000003-2947-8641; S. Scarsoglio, https://orcid.org/0000-0002-9427-6491

\section{REFERENCES}

Agostini, L. \& LeschZiner, M. 2014 On the influence of outer large-scale structures on near-wall turbulence in channel flow. Physics of Fluids 26 (7), 075107. 
Agostini, L. \& Leschziner, M. 2016 Predicting the response of small-scale near-wall turbulence to largescale outer motions. Physics of Fluids 28 (1), 015107.

Agostini, L. \& LeschZiner, M. 2019 On the departure of near-wall turbulence from the quasi-steady state. J. Fluid Mech. 871 (R1).

Agostini, L., Leschziner, M. \& Gaitonde, D. 2016 Skewness-induced asymmetric modulation of smallscale turbulence by large-scale structures. Physics of Fluids 28 (1), 015110.

Anderson, W. 2016 Amplitude modulation of streamwise velocity fluctuations in the roughness sublayer: evidence from large-eddy simulations. Journal of Fluid Mechanics 789, 567-588.

Awasthi, A. \& Anderson, W. 2018 Numerical study of turbulent channel flow perturbed by spanwise topographic heterogeneity: amplitude and frequency modulation within low-and high-momentum pathways. Physical Review Fluids 3 (4), 044602.

BAARs, W.J., Hutchins, N. \& Marusic, I. 2017 Reynolds number trend of hierarchies and scale interactions in turbulent boundary layers. Philosophical Transactions of the Royal Society A 375 (2089), 20160077.

Baars, W.J., Talluru, K.M., Hutchins, N. \& Marusic, I. 2015 Wavelet analysis of wall turbulence to study large-scale modulation of small scales. Experiments in fluids $\mathbf{5 6}$ (10), 188.

BaArs, W. J., Hutchins, N. \& Marusic, I. 2016 Spectral stochastic estimation of high-Reynolds-number wall-bounded turbulence for a refined inner-outer interaction model. Physical Review Fluids 1 (5), 054406 .

BALAKUMAR, B.J. \& AdRian, R.J. 2007 Large-and very-large-scale motions in channel and boundary-layer flows. Philosophical Transactions of the Royal Society A 365 (1852), 665-681.

Bandyopadhyay, P. R. \& Hussain, A.K.M.F. 1984 The coupling between scales in shear flows. The Physics of Fluids 27 (9), 2221-2228.

Basley, J., Perret, L. \& Mathis, R. 2018 Spatial modulations of kinetic energy in the roughness sublayer. Journal of Fluid Mechanics 850, 584-610.

Bernardini, M. \& Pirozzoli, S. 2011 Inner/outer layer interactions in turbulent boundary layers: a refined measure for the large-scale amplitude modulation mechanism. Physics of Fluids $\mathbf{2 3}$ (6), 061701.

Bonshash, B. 2015 Time-frequency signal analysis and processing: a comprehensive reference. Academic Press.

Brown, G. L. \& Thomas, A. S.W. 1977 Large structure in a turbulent boundary layer. The Physics of Fluids 20 (10), S243-S252.

Charakopoulos, A.K., Karakasidis, T.E., Papanicolaou, P.N. \& Liakopoulos, A. 2014 The application of complex network time series analysis in turbulent heated jets. Chaos 24 (2), 024408.

Chernyshenko, S. 2020 Extension of QSQH theory of scale interaction in near-wall turbulence to all velocity components. arXiv preprint 2002.05585 .

Chung, D. \& McKeon, B.J. 2010 Large-eddy simulation of large-scale structures in long channel flow. Journal of Fluid Mechanics 661, 341-364.

Dogan, E., Örlü, R., Gatti, D., Vinuesa, R. \& Schlatter, P. 2019 Quantification of amplitude modulation in wall-bounded turbulence. Fluid Dynamics Research 51 (1), 011408.

Duvvuri, S. \& McKeon, B. J. 2015 Triadic scale interactions in a turbulent boundary layer. Journal of Fluid Mechanics $\mathbf{7 6 7 .}$

Fiscaletti, D., Attili, A., Bisetti, F. \& Elsinga, G. E. 2016 Scale interactions in a mixing layer - the role of the large-scale gradients. Journal of Fluid Mechanics 791, 154-173.

Fiscaletti, D., Ganapathisubramani, B. \& Elsinga, G.E. 2015 Amplitude and frequency modulation of the small scales in a jet. Journal of Fluid Mechanics 772, 756-783.

Ganapathisubramani, B., Hutchins, N., Monty, J.P., Chung, D. \& Marusic, I. 2012 Amplitude and frequency modulation in wall turbulence. Journal of Fluid Mechanics 712 (61), 064602-17.

Graham, J., Kanov, K., Yang, X., Lee, M., Malaya, N., Lalescu, C.C., Burns, R., Eyink, G., Szalay, A., Moser, R.D. \& Meneveau, C. 2016 A web services accessible database of turbulent channel flow and its use for testing a new integral wall model for LES. Journal of Turbulence 17 (2), 181-215.

Guala, M., Metzger, M. \& McKeon, B. J. 2011 Interactions within the turbulent boundary layer at high Reynolds number. Journal of Fluid Mechanics 666, 573-604.

Hasson, U., Iacovacci, J., Davis, B., Flanagan, R., Tagliazucchi, E., Laufs, H. \& Lacasa, L. 2018 A combinatorial framework to quantify peak/pit asymmetries in complex dynamics. Scientific reports $8(1), 1-17$.

Hu, R. \& ZhENG, X. 2018 Energy contributions by inner and outer motions in turbulent channel flows. Physical Review Fluids 3 (8), 084607. 
Huang, N. E., Shen, Z., Long, S. R., Wu, M. C., Shin, H. H., Zheng, Q., Yen, N.C., Tung, C. C. \& Liu, H. H. 1998 The empirical mode decomposition and the hilbert spectrum for nonlinear and non-stationary time series analysis. Proceedings of the Royal Society of London A 454 (1971), 903-995.

Hutchins, N. 2014 Large-scale structures in high Reynolds number wall-bounded turbulence. In Progress in turbulence $V$ - iTi 2018, pp. 75-83. Springer.

Hutchins, N. \& Marusic, I. 2007a Evidence of very long meandering features in the logarithmic region of turbulent boundary layers. Journal of Fluid Mechanics 579, 1-28.

Hutchins, N. \& Marusic, I. $2007 b$ Large-scale influences in near-wall turbulence. Philosophical Transactions of the Royal Society A 365 (1852), 647-664.

Hwang, J., Lee, J., Sung, H. J. \& Zaki, T. A. 2016 Inner-outer interactions of large-scale structures in turbulent channel flow. Journal of Fluid Mechanics 790, 128-157.

IACoBello, G. 2020 Fast natural visibility graph (NVG) for MATLAB. MATLAB Central File Exchange.

Iacobello, G., Marro, M., Ridolfi, L., Salizzoni, P. \& Scarsoglio, S. $2019 a$ Experimental investigation of vertical turbulent transport of a passive scalar in a boundary layer: Statistics and visibility graph analysis. Physical Review Fluids 4 (10), 104501.

Iacobello, G., Ridolfi, L., Marro, M., Salizzoni, P. \& Scarsoglio, S. 2018 a Complex network analysis of wind tunnel experiments on the passive scalar dispersion in a turbulent boundary layer. In Progress in Turbulence VIII - iTi 2018, pp. 215-220. Springer.

Iacobello, G., Ridolfi, L. \& Scarsoglio, S. 2021 A review on turbulent and vortical flow analyses via complex networks. Physica A 563, 125476.

Iacobello, G., Scarsoglio, S., Kuerten, J.G.M. \& Ridolfi, L. $2019 b$ Lagrangian network analysis of turbulent mixing. Journal of Fluid Mechanics 865, 546-562.

Iacobello, G., Scarsoglio, S. \& Ridolfi, L. $2018 b$ Visibility graph analysis of wall turbulence time-series. Physics Letters A 382 (1), 1-11.

JiMÉNEZ, J. 2018 Coherent structures in wall-bounded turbulence. Journal of Fluid Mechanics $\mathbf{8 4 2}$.

Krishnan, A., Sujith, R.I., Marwan, N. \& Kurths, J. 2019 On the emergence of large clusters of acoustic power sources at the onset of thermoacoustic instability in a turbulent combustor. Journal of Fluid Mechanics 874, 455-482.

Lacasa, L. \& Iacovacci, J. 2017 Visibility graphs of random scalar fields and spatial data. Physical Review E 96 (1), 012318.

Lacasa, L., Luque, B., Ballesteros, F., Luque, J. \& Nuno, J.C. 2008 From time series to complex networks: The visibility graph. Proceedings of the National Academy of Sciences 105 (13), 49724975.

Lee, My. \& Moser, R. D. 2015 Direct numerical simulation of turbulent channel flow up to $R e_{\tau} \approx 5200$. Journal of Fluid Mechanics 774, 395-415.

Li, Y., Perlman, E., Wan, M., Yang, Y., Meneveau, C., Burns, R., Chen, S., Szalay, A. \& Eyink, G. 2008 A public turbulence database cluster and applications to study lagrangian evolution of velocity increments in turbulence. Journal of Turbulence $\mathbf{9}$, N31.

Liv, C., Zhou, W.X. \& YuAN, W.K. 2010 Statistical properties of visibility graph of energy dissipation rates in three-dimensional fully developed turbulence. Physica A 389 (13), 2675-2681.

Luque, B., Lacasa, L., Ballesteros, F. \& Luque, J. 2009 Horizontal visibility graphs: Exact results for random time series. Physical Review E 80 (4), 046103.

Marusic, I. 2020 Two-point high Reynolds number zero-pressure gradient turbulent boundary layer dataset DOI: $10.26188 / 5 \mathrm{e} 919 \mathrm{e} 62 \mathrm{e} 0 \mathrm{dac}$.

Marusic, I. \& Heuer, W. D.C. 2007 Reynolds number invariance of the structure inclination angle in wall turbulence. Physical Review Letters 99 (11), 114504.

Marusic, I., Mathis, R. \& Hutchins, N. 2010 Predictive model for wall-bounded turbulent flow. Science 329 (5988), 193-196.

Mathis, R., Hutchins, N. \& Marusic, I. 2009a Large-scale amplitude modulation of the small-scale structures in turbulent boundary layers. Journal of Fluid Mechanics 628, 311-337.

Mathis, R., Hutchins, N. \& Marusic, I. 2011 A predictive inner-outer model for streamwise turbulence statistics in wall-bounded flows. Journal of Fluid Mechanics 681, 537-566.

Mathis, R., Marusic, I., Chernyshenko, S. I. \& Hutchins, N. 2013 Estimating wall-shear-stress fluctuations given an outer region input. Journal of Fluid Mechanics 715, 163.

Mathis, R., Monty, J. P., Hutchins, N. \& Marusic, I. $2009 \mathrm{~b}$ Comparison of large-scale amplitude modulation in turbulent boundary layers, pipes, and channel flows. Physics of Fluids 21 (11), 111703. 
Monty, J.P., Hutchins, N., NG, H.C.H., Marusic, I. \& Chong, M.S. 2009 A comparison of turbulent pipe, channel and boundary layer flows. Journal of Fluid Mechanics 632, 431-442.

Monty, J.P., Stewart, J.A., Williams, R.C. \& Chong, M.S. 2007 Large-scale features in turbulent pipe and channel flows. Journal of Fluid Mechanics 589, 147.

Murugesan, M. \& Sujith, R.I. 2015 Combustion noise is scale-free: transition from scale-free to order at the onset of thermoacoustic instability. Journal of Fluid Mechanics 772, 225-245.

Murugesan, M. \& Sujith, R.I. 2016 Detecting the onset of an impending thermoacoustic instability using complex networks. Journal of Propulsion and Power 32 (3), 707-712.

Murugesan, M., Zhu, Y. \& Li, L. K.B. 2019 Complex network analysis of forced synchronization in a hydrodynamically self-excited jet. International Journal of Heat and Fluid Flow 76, 14-25.

Newman, M. 2018 Networks, 2nd edn. Oxford University Press.

Pathikonda, G. \& Christensen, K. T. 2017 Inner-outer interactions in a turbulent boundary layer overlying complex roughness. Physical Review Fluids 2 (4), 044603.

Pathikonda, G. \& Christensen, K. T. 2019 Investigation of inner-outer interactions in a turbulent boundary layer using high-speed particle image velocimetry. Physical Review Fluids 4 (3), 034607.

Peruzzi, C., Poggi, D., Ridolfi, L. \& Manes, C. 2020 On the scaling of large-scale structures in smooth-bed turbulent open-channel flows. Journal of Fluid Mechanics $\mathbf{8 8 9}$.

SCHLATTER, P. \& ÖRL ̈̈, R. 2010 Quantifying the interaction between large and small scales in wall-bounded turbulent flows: a note of caution. Physics of fluids 22 (5), 051704.

Schlueter-Kuck, K. L. \& Dabiri, J. O. 2017 Coherent structure colouring: identification of coherent structures from sparse data using graph theory. Journal of Fluid Mechanics 811, 468-486.

Singh, J., Belur Vishwanath, R., Chaudhuri, S. \& Sujith, R.I. 2017 Network structure of turbulent premixed flames. Chaos 27 (4), 043107.

Smits, A. J., McKeon, B. J. \& Marusic, I. 2011 High-Reynolds number wall turbulence. Annual Review of Fluid Mechanics 43.

Squire, D.T., BaArs, W.J., Hutchins, N. \& Marusic, I. 2016 Inner-outer interactions in rough-wall turbulence. Journal of Turbulence 17 (12), 1159-1178.

Taira, K., Nair, A.G. \& Brunton, S.L. 2016 Network structure of two-dimensional decaying isotropic turbulence. Journal of Fluid Mechanics 795, R2.

Talluru, K.M., Baidya, R., Hutchins, N. \& Marusic, I. 2014 Amplitude modulation of all three velocity components in turbulent boundary layers. Journal of Fluid Mechanics $\mathbf{7 4 6 .}$

TANG, Z. \& JIANG, N. 2018 Scale interaction and arrangement in a turbulent boundary layer perturbed by a wall-mounted cylindrical element. Physics of Fluids 30 (5), 055103.

Tokami, T., Hachijo, T., Miyano, T. \& Gotoda, H. 2020 Spatiotemporal dynamics of a buoyancy-driven turbulent fire. Physical Review E 101 (4), 042214.

Wu, S., Christensen, K. T. \& Pantano, C. 2019 Modelling smooth-and transitionally rough-wall turbulent channel flow by leveraging inner-outer interactions and principal component analysis. Journal of Fluid Mechanics 863, 407-453.

YANG, X.I.A. \& Howland, M.F. 2018 Implication of Taylor's hypothesis on measuring flow modulation. Journal of Fluid Mechanics 836, 222.

Yao, Y.C., Huang, W.X. \& Xu, C.X. 2018 Amplitude modulation and extreme events in turbulent channel flow. Acta Mechanica Sinica 34 (1), 1-9.

ZhANG, C. \& CHERnYShenko, S. I. 2016 Quasisteady quasihomogeneous description of the scale interactions in near-wall turbulence. Physical Review Fluids 1 (1), 014401.

Zhuang, E., Small, M. \& Feng, G. 2014 Time series analysis of the developed financial markets' integration using visibility graphs. Physica A 410, 483-495.

Zou, Y., Donner, R. V., Marwan, N., Donges, J. F. \& Kurths, J. 2018 Complex network approaches to nonlinear time series analysis. Physics Reports 787, 1-97. 